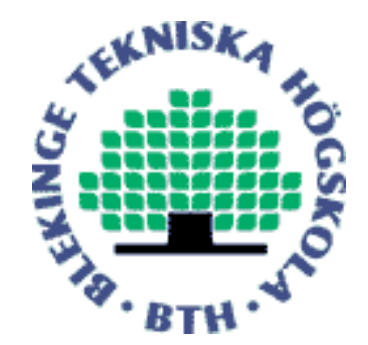

Copyright @ 2013 IEEE.

Citation for the published paper:

Title:

In press: Uplink Performance Analysis of Multicell MU-SIMO Systems with ZF receivers

Author:

Hien Quoc Ngo, Michail Matthaiou, Quang Trung Duong, Erik G. Larsson

Journal:

IEEE Trans on Vehicular Technology

Year:

2013

Vol:

Issue:

Pagination:

URL/DOI to the paper:

10.1109/TVT.2013.2265720

This material is posted here with permission of the IEEE. Such permission of the IEEE does not in any way imply IEEE endorsement of any of BTH's products or services Internal or personal use of this material is permitted. However, permission to reprint/republish this material for advertising or promotional purposes or for creating new collective works for resale or redistribution must be obtained from the IEEE by sending a blank email message to pubs-permissions@iee.org.

By choosing to view this document, you agree to all provisions of the copyright laws protecting it. 


\title{
Uplink Performance Analysis of Multicell MU-SIMO Systems with ZF Receivers
}

\author{
Hien Quoc Ngo, Student Member, IEEE, Michail Matthaiou, Member, IEEE, \\ Trung Q. Duong, Senior Member, IEEE, and Erik G. Larsson, Senior Member, IEEE
}

\begin{abstract}
We consider the uplink of a multicell multiuser single-input multiple-output system, where the channel experiences both small and large-scale fading. The data detection is done by using the linear zero-forcing technique, assuming the base station (BS) has perfect channel state information of all users in its cell. We derive new, exact analytical expressions for the uplink rate, symbol error rate, and outage probability per user, as well as a lower bound on the achievable rate. This bound is very tight and becomes exact in the large-number-of-antennas limit. We further study the asymptotic system performance in the regimes of high signal-to-noise ratio (SNR), large number of antennas, and large number of users per cell. We show that at high SNRs, the system is interference-limited and hence, we cannot improve the system performance by increasing the transmit power of each user. Instead, by increasing the number of BS antennas, the effects of interference and noise can be reduced, thereby improving the system performance. We demonstrate that, with very large antenna arrays at the $B S$, the transmit power of each user can be made inversely proportional to the number of BS antennas while maintaining a desired quality-of-service. Numerical results are presented to verify our analysis.
\end{abstract}

Index Terms-Multiuser SIMO, very large MIMO systems, zero-forcing receiver.

\section{INTRODUCTION}

$\mathbf{M}$ ULTIPLE-INPUT multiple-output (MIMO) technology can provide a remarkable increase in data rate and reliability compared to single-antenna systems. Recently, multiuser MIMO (MU-MIMO) configurations, where the base stations (BSs) are equipped with multiple antennas and communicate with several co-channel users, have gained much attention and are now being introduced in several new generation wireless standards (e.g., LTE-Advanced, 802.16m) [2]. This scheme is

Copyright (c) 2013 IEEE. Personal use of this material is permitted. However, permission to use this material for any other purposes must be obtained from the IEEE by sending a request to pubs-permissions@ieee.org.

Manuscript received October 19, 2012; revised March 21, 2013; accepted May 21, 2013. The associate editor coordinating the review of this paper and approving it for publication was Sami Muhaidat.

H. Q. Ngo and E. G. Larsson are with the Department of Electrical Engineering (ISY), Linköping University, 58183 Linköping, Sweden (email: nqhien@isy.liu.se; egl@isy.liu.se).

M. Matthaiou is with the Department of Signals and Systems, Chalmers University of Technology, 41296 Gothenburg, Sweden (email: michail.matthaiou@chalmers.se).

T. Q. Duong is with the Blekinge Institute of Technology, 37179 Karlskrona, Sweden (email: quang.trung.duong@bth.se).

The work of H. Q. Ngo and E. G. Larsson was supported in part by the Swedish Research Council (VR), the Swedish Foundation for Strategic Research (SSF), and ELLIIT. The work of M. Matthaiou was supported in part by the Swedish Governmental Agency for Innovation Systems (VINNOVA) within the VINN Excellence Center Chase. Parts of this work were presented at the 2011 IEEE Swedish Communication Technologies Workshop [1].

Digital Object Identifier $\mathrm{xxx} / \mathrm{xxx}$ also known as space division multiple access (SDMA), which provides high bandwidth efficiency and higher throughput than time division multiple access. The goal of the SDMA scheme is to improve the cell capacity (more users are simultaneously served), while keeping the spectrum allocation unchanged. SDMA normally requires that the number of BS antennas is larger than the number of users that share the same spectrum. In the uplink, the BS is able to decode the signal transmitted from each user, while avoiding the signals transmitted from the other users. The optimal SDMA scheme for the uplink is joint multiuser detection. However, it is too complex for practical implementation. More practical SDMA detection algorithms are based on linear processing, including zero-forcing (ZF) or minimum mean-square error (MMSE) [3].

MU-MIMO systems have been studied from many perspectives including communication, signalling, and information theory in both downlink and uplink scenarios [4]-[6]. All these mentioned works have only investigated a single-cell scenario, where the effects of intercell interference have been neglected. However, co-channel interference, appearing due to frequencyreuse, represents an important impairment in cellular systems. Recently, there has been an increasing research interest in the performance of MU-MIMO systems in interference-limited multicell environments [7]-[11]. In fact, it has been shown that the capacity of the MU-MIMO downlink can be dramatically reduced due to intercell interference [7].

Many interference cancellation and mitigation techniques have been proposed for multicell MU-MIMO systems, such as maximum likelihood multiuser detection [9], [12], BS cooperation [13], and interference alignment [14]. These techniques, however, induce a significant complexity burden on the system implementation, especially for large array configurations. Therefore, linear receivers/precoders, in particular $\mathrm{ZF}$, are of particular interest as low-complexity alternatives [15]-[18]. When the number of BS antennas is small, linear receivers/precoders do not perform well due to interuser interference. But when the number of BS antennas is large, the channel vectors are nearly orthogonal and hence, interference can be successfully handled by using simple linear receivers/precoders. As a consequence, with very large antenna arrays, optimal performance can be achieved even with simple linear processing, like ZF (see e.g., [19], [20] for a more detailed discussion). Very recently, there has been a great deal of interest in multicell MU-MIMO systems, where several BSs are equipped with very large antenna arrays [19]-[23]. In this context, the asymptotic signal-to-interference-plus-noise ratios (SINRs), when the number of BS antennas grows to 
infinity, were derived in [21] for maximum-ratio combining (MRC) in the uplink and maximum-ratio transmission in the downlink. In [23], using tools of random matrix theory, the authors derived a deterministic approximation of the uplink SINR with MRC and MMSE receivers, assuming that the number of transmit antennas and number of users go to infinity at the same rate. They also showed that the deterministic approximation of the SINR is tight even with a moderate number of BS antennas and users. However, since the limiting SINR obtained therein is deterministic, this approximation does not enable us to further analyze other figures of merit, such as the outage probability or symbol error rate (SER). More importantly, iterative algorithms are needed to compute the deterministic equivalent results. In [20], lower bounds on the uplink achievable rates with linear detectors were computed, and the authors showed that MRC performs as well as ZF in a regime where the spectral efficiency is of the order of 1 bit per channel use per user. Nevertheless, it was demonstrated that ZF performs much better than MRC at higher spectral efficiencies.

Inspired by the above discussion, in this paper, we analyze the performance of multicell multiuser single-input multipleoutput (MU-SIMO) systems, where many single-antenna users simultaneously transmit data to a BS. The BS uses ZF to detect the transmitted signals. Note that the MMSE receiver always performs better than the ZF receiver. However, herein we consider ZF receivers for the following reasons: i) an exact analysis of MMSE receivers is a challenging mathematical problem in a multicell MU-SIMO setup. This implication can be seen by invoking the generic results of [24]; ii) the implementation of MMSE requires additional knowledge of the noise and interference statistics; iii) it is well-known that ZF receivers perform equivalently to MMSE receivers at high SINRs [25]; and iv) the performance of ZF bounds that of MMSE from below, so the results we obtain represent achievable lower bounds on the MMSE receivers' performance. The paper makes the following specific contributions: ${ }^{1}$

- We derive exact analytical expressions for the ergodic data rate, SER, and outage probability of the uplink channel for any finite number of BS antennas. We also derive a tractable lower bound on the achievable rate. Note that, although these exact results involve complicated functions, they can be more efficiently evaluated compared to brute-force Monte-Carlo simulations, especially for large configurations.

- Next, we focus on the ZF receiver's asymptotic performance, when the BS deploys a large antenna array. These results enable us to explicitly study the effects of transmit power, intercell interference, and number of BS antennas. For instance, when the number of users per cell is fixed and the number of BS antennas grows without bound, intercell interference and noise are averaged out. However, when fixing the ratio between the number of BS

\footnotetext{
${ }^{1}$ The work presented here is a comprehensive extension of our conference paper [1]. The main novel elements over [1] are: i) a new tractable lower bound on the achievable rate; ii) an analysis of SER and outage probability; and iii) asymptotic system analysis in the regime where the number of transmit antennas and number of users go to infinity with a fixed, finite ratio.
}

antennas and the number of users, intercell interference does not vanish when the number of antennas grows large. Yet, in both cases by using very large antenna arrays, the transmit power of each user can be made inversely proportional to the number of antennas with no performance degradation.

Notation: The superscript $H$ stands for conjugate transpose, while $[\boldsymbol{A}]_{i j}$ denotes the $(i, j)$ th entry of a matrix $\boldsymbol{A}$, and $\boldsymbol{I}_{n}$ is the $n \times n$ identity matrix. The expectation operation, the Euclidean norm, and the trace operator are denoted by $\mathbb{E}\{\cdot\}$, $\|\cdot\|$, and $\operatorname{Tr}(\cdot)$, respectively. The notation $\stackrel{\text { a.s. }}{\rightarrow}$ means almost sure convergence. We use $a \stackrel{d}{\sim} b$ to imply that $a$ and $b$ have the same distribution. Finally, we use $\boldsymbol{z} \sim \mathcal{C N}(\mathbf{0}, \boldsymbol{\Sigma})$ to denote a circularly symmetric complex Gaussian vector $z$ with zeromean and covariance matrix $\boldsymbol{\Sigma}$.

\section{Multicell MU-Simo System}

In the following, we consider a multicell MU-SIMO system with $L$ cells. Each cell includes one BS equipped with $N$ antennas, and $K$ single-antenna users $(N \geq K)$. We consider uplink transmission, and assume that the $L$ BSs share the same frequency band. Conventionally, the communication between the BS and the users is performed in separate time-frequency resources. However, when the BS is equipped with more antennas, more degrees of freedom are offered and hence, more independent data streams can be transmitted. Therefore, it is more efficient if several users communicate with the BS in the same time-frequency resource [11], [21]. We assume that all users simultaneously transmit data streams to their BSs. ${ }^{2}$ The $N \times 1$ received vector at the $l$ th $\mathrm{BS}(l=1, \ldots, L)$ is

$$
\boldsymbol{y}_{l}=\sqrt{p_{\mathrm{u}}} \sum_{i=1}^{L} \boldsymbol{G}_{l i} \boldsymbol{x}_{i}+\boldsymbol{n}_{l}
$$

where $\boldsymbol{G}_{l i} \in \mathbb{C}^{N \times K}$ is the channel matrix between the lth BS and the $K$ users in the $i$ th cell, i.e., $g_{l i m k} \triangleq\left[\boldsymbol{G}_{l i}\right]_{m k}$ is the channel coefficient between the $m$ th antenna of the $l$ th BS and the $k$ th user in the $i$ th cell; $\sqrt{p_{\mathrm{u}}} \boldsymbol{x}_{i} \in \mathbb{C}^{K \times 1}$ is the transmitted vector of $K$ users in the $i$ th cell (the average power transmitted by each user is $p_{\mathrm{u}}$ ); and $\boldsymbol{n}_{l} \in \mathbb{C}^{N \times 1}$ is an additive white Gaussian noise (AWGN) vector, such that $\boldsymbol{n}_{l} \sim \mathcal{C N}\left(\mathbf{0}, \boldsymbol{I}_{M}\right)$. Note that, since the noise power is assumed to be $1, p_{\mathrm{u}}$ can be considered as the normalized "transmit" SNR and hence, it is dimensionless. Here, we assume equal transmit power for all users. This assumption does not affect our analytical methodologies and the obtained results, and can provide a lower bound on the performance of practical systems, where power control is being used.

The channel matrix, $\boldsymbol{G}_{l i}$, models independent small-scale fading, path-loss attenuation, and lognormal shadow fading. The assumption of independent small-scale fading is sufficiently realistic for systems where the antennas are sufficiently

\footnotetext{
${ }^{2}$ It arguably would be more practical to consider asynchronous transmission. Unfortunately, if we consider the impact of asynchronous transmission, the system model becomes too complicated for analysis. Note that our synchronous-transmission results can be regarded as an upper bound of what is actually achieved in practice [26].
} 
well separated [27]. The channel coefficient $g_{\text {limk }}$ is given by

$$
g_{\text {limk }}=h_{\text {limk }} \sqrt{\beta_{\text {lik }}}, \quad m=1,2, \ldots, N
$$

where $h_{\text {limk }}$ is the small-scale fading coefficient from the $k$ th user in the $i$ th cell to the $m$ th antenna of the $l$ th BS. The coefficient $h_{\text {limk }}$ is assumed to be complex Gaussian distributed with zero-mean and unit variance. Moreover, $\sqrt{\beta_{l i k}}$ represents the path-loss attenuation and shadow fading, which are assumed to be constant over the index of the BS antenna, $m$, and over many coherence intervals. This assumption is reasonable for a collocated BS antenna array since the distance between users and the BS is much greater than the distance between the BS antennas. More importantly, the validity of this assumption has been demonstrated in practice even for large antenna arrays [28].

We assume that the BS has perfect channel state information (CSI) of all users in its cell. This assumption is reasonable in an environment with low or moderate mobility, so that long training intervals can be afforded. ${ }^{3}$ Moreover, the results obtained under this assumption serve as bounds on the performance for the case that CSI is imperfect due to estimation errors or feedback delay. We further assume that the transmitted signals from the $K$ users in the $l$ th cell are detected using a ZF receiver. As such, the received vector $\boldsymbol{y}_{l}$ is processed by multiplying it with the pseudo-inverse of $\boldsymbol{G}_{l l}$ as:

$$
\boldsymbol{r}_{l}=\boldsymbol{G}_{l l}^{\dagger} \boldsymbol{y}_{l}=\sqrt{p_{\mathrm{u}}} \boldsymbol{x}_{l}+\sqrt{p_{\mathrm{u}}} \sum_{i \neq l}^{L} \boldsymbol{G}_{l l}^{\dagger} \boldsymbol{G}_{l i} \boldsymbol{x}_{i}+\boldsymbol{G}_{l l}^{\dagger} \boldsymbol{n}_{l}
$$

where $\boldsymbol{G}_{l l}^{\dagger} \triangleq\left(\boldsymbol{G}_{l l}^{H} \boldsymbol{G}_{l l}\right)^{-1} \boldsymbol{G}_{l l}^{H}$. Therefore, the $k$ th element of $r_{l}$ is given by

$$
\boldsymbol{r}_{l, k}=\sqrt{p_{\mathrm{u}}} \boldsymbol{x}_{l, k}+\sqrt{p_{\mathrm{u}}} \sum_{i \neq l}^{L}\left[\boldsymbol{G}_{l l}^{\dagger}\right]_{k} \boldsymbol{G}_{l i} \boldsymbol{x}_{i}+\left[\boldsymbol{G}_{l l}^{\dagger}\right]_{k} \boldsymbol{n}_{l}
$$

where $\boldsymbol{x}_{l, k}$ is the $k$ th element of $\boldsymbol{x}_{l}$, which is the transmitted signal from the $k$ th user in the $l$ th cell, while $[\boldsymbol{A}]_{k}$ denotes the $k$ th row of a matrix $\boldsymbol{A}$. Note that since we use ZF receivers, intracell interference is completely canceled out. From (4), the SINR of the uplink transmission from the $k$ th user in the $l$ th cell to its BS is defined as

$$
\gamma_{k} \triangleq \frac{p_{\mathrm{u}}}{p_{\mathrm{u}} \sum_{i \neq l}^{L}\left\|\left[\boldsymbol{G}_{l l}^{\dagger}\right]_{k} \boldsymbol{G}_{l i}\right\|^{2}+\left\|\left[\boldsymbol{G}_{l l}^{\dagger}\right]_{k}\right\|^{2}} .
$$

Proposition 1: The SINR of the uplink transmission from the $k$ th user in the $l$ th cell to its BS can be represented as

$$
\gamma_{k} \stackrel{\mathrm{d}}{\sim} \frac{p_{\mathrm{u}} X_{k}}{p_{\mathrm{u}} Z_{l}+1}
$$

\footnotetext{
${ }^{3}$ In multiuser systems with very large antenna arrays at the BS, a standard way to obtain the CSI is to use uplink pilots. If the coherence interval is short, non-orthogonal pilot sequences must be utilized in different cells. As a result, the channel estimate in a given cell is contaminated by the pilots transmitted from users in other cells. This effect is known as "pilot contamination" [11]. By contrast, here, we assume that the coherence interval is long enough so that all cells are assigned orthogonal pilot sequences and hence, the pilot contamination effect disappears.
}

where $X_{k}$ and $Z_{l}$ are independent RVs whose probability density functions (PDFs) are respectively given by

$$
\begin{aligned}
p_{X_{k}}(x) & =\frac{e^{-x / \beta_{l l k}}}{(N-K) ! \beta_{l l k}}\left(\frac{x}{\beta_{l l k}}\right)^{N-K}, x \geq 0 \\
p_{Z_{l}}(z) & =\sum_{m=1}^{\varrho\left(\mathcal{A}_{l}\right) \tau_{m}\left(\mathcal{A}_{l}\right)} \sum_{n=1} \mathcal{X}_{m, n}\left(\mathcal{A}_{l}\right) \frac{\mu_{l, m}^{-n}}{(n-1) !} z^{n-1} e^{\frac{-z}{\mu_{l, m}}}, z \geq 0
\end{aligned}
$$

where $\mathcal{A}_{l} \in \mathbb{C}^{K(L-1) \times K(L-1)}$ is given by

$$
\mathcal{A}_{l} \triangleq\left[\begin{array}{cccccc}
\boldsymbol{D}_{l 1} & & & & & \\
& \ddots & & & 0 & \\
& & \boldsymbol{D}_{l(l-1)} & & & \\
& & & \boldsymbol{D}_{l(l+1)} & & \\
& \mathbf{0} & & & \ddots & \boldsymbol{D}_{l L}
\end{array}\right]
$$

with $\boldsymbol{D}_{l i}$ is a $K \times K$ diagonal matrix whose elements are given by $\left[\boldsymbol{D}_{l i}\right]_{k k}=\beta_{l i k} ; \varrho\left(\mathcal{A}_{l}\right)$ is the number of distinct diagonal elements of $\mathcal{A}_{l} ; \mu_{l, 1}, \mu_{l, 2}, \ldots, \mu_{l, \varrho\left(\mathcal{A}_{l}\right)}$ are the distinct diagonal elements in decreasing order; $\tau_{m}\left(\mathcal{A}_{l}\right)$ is the multiplicity of $\mu_{l, m}$; and $\mathcal{X}_{m, n}\left(\mathcal{A}_{l}\right)$ is the $(m, n)$ th characteristic coefficient of $\mathcal{A}_{l}$ which is defined in [31, Definition 4].

Proof: Dividing the denominator and numerator of (5) by $\left\|\left[\boldsymbol{G}_{l l}^{\dagger}\right]_{k}\right\|^{2}$, we obtain

$$
\gamma_{k}=\frac{p_{\mathrm{u}}\left\|\left[\boldsymbol{G}_{l l}^{\dagger}\right]_{k}\right\|^{-2}}{p_{\mathrm{u}} \sum_{i \neq l}^{L}\left\|\boldsymbol{Y}_{i}\right\|^{2}+1}
$$

where $\boldsymbol{Y}_{i} \triangleq \frac{\left[\boldsymbol{G}_{l l}^{\dagger}\right]_{k} \boldsymbol{G}_{l i}}{\left\|\left[\boldsymbol{G}_{l l}^{\dagger}\right]_{k}\right\|}$. Since $\left\|\left[\boldsymbol{G}_{l l}^{\dagger}\right]_{k}\right\|^{2}=\left[\left(\boldsymbol{G}_{l l}^{H} \boldsymbol{G}_{l l}\right)^{-1}\right]_{k k}$, $\left\|\left[\boldsymbol{G}_{l l}^{\dagger}\right]_{k}\right\|^{-2}$ has an Erlang distribution with shape parameter $N-K^{k}+1$ and scale parameter $\beta_{l l k}$ [32], then

$$
\left\|\left[\boldsymbol{G}_{l l}^{\dagger}\right]_{k}\right\|^{-2} \stackrel{\mathrm{d}}{\sim} X_{k} .
$$

We next show that $\boldsymbol{Y}_{i}$ and $X_{k}$ are independent. Conditioned on $\left[\boldsymbol{G}_{l l}^{\dagger}\right]_{k}, \boldsymbol{Y}_{i}$ is a zero-mean complex Gaussian vector with covariance matrix $\boldsymbol{D}_{l i}$ which is independent of $\left[\boldsymbol{G}_{l l}^{\dagger}\right]_{k}$. Since the PDF of a Gaussian vector is fully described via its first and second moments, $\boldsymbol{Y}_{i}$ is a Gaussian vector which is independent of $\left[\boldsymbol{G}_{l l}^{\dagger}\right]_{k}$ and, in turn, of $X_{k}$. Then, $\sum_{i \neq l}^{L}\left\|\boldsymbol{Y}_{i}\right\|^{2}$ is independent of $X_{k}^{k}$, and is the sum of $K(L-1)$ statistically independent but not necessarily identically distributed exponential RVs. Thus, from [33, Theorem 2], we have that

$$
\sum_{i \neq l}^{L}\left\|Y_{i}\right\|^{2} \stackrel{\mathrm{d}}{\sim} Z_{l}
$$

From (9)-(11), we can obtain (6).

\section{Finite- $N$ ANALYSis}

In this section, we present exact analytical expressions for the ergodic uplink rate, SER, and outage probability of the system described in Section II. We underline the fact that the following results hold for any arbitrary number of BS antennas, provided that $N \geq K$. 
This article has been accepted for publication in a future issue of this journal, but has not been fully edited. Content may change prior to final publication.

$$
\begin{array}{r}
\left\langle R_{l, k}\right\rangle=\log _{2} e \sum_{m=1}^{\varrho\left(\mathcal{A}_{l}\right)} \sum_{n=1}^{\tau_{m}\left(\mathcal{A}_{l}\right)} \sum_{p=0}^{N-K} \frac{\mathcal{X}_{m, n}\left(\mathcal{A}_{l}\right) \mu_{l, m}^{-n}(-1)^{N-K-p}}{(n-1) !(N-K-p) !}\left[-e^{\frac{1}{\beta_{l l k} p_{\mathrm{u}}}} \mathcal{I}_{n-1, N-K-p}\left(\frac{1}{\beta_{l l k}}, \frac{1}{\beta_{l l k} p_{\mathrm{u}}}, \frac{1}{\mu_{l, m}}-\frac{1}{\beta_{l l k}}\right)\right. \\
\left.\quad+\sum_{q=1}^{N-K-p} \frac{(q-1) !(-1)^{q} p_{\mathrm{u}}^{-n}}{\left(\beta_{l l k} p_{\mathrm{u}}\right)^{N-K-p-q}} \Gamma(n) U\left(n, n+N+1-K-p-q, \frac{1}{\mu_{l, m} p_{\mathrm{u}}}\right)\right]
\end{array}
$$

$$
\begin{aligned}
& \mathcal{I}_{m, n}(a, b, \alpha) \triangleq \sum_{i=0}^{m}\left(\begin{array}{c}
m \\
i
\end{array}\right)(-b)^{m-i}\left[\sum_{q=0}^{n+i} \frac{(n+i)^{q} b^{n+i-q}}{\alpha^{q+1} a^{m-q}}\right. \operatorname{Ei}(-b)-\frac{(n+i)^{n+i} e^{\alpha b / a}}{\alpha^{n+i+1} a^{m-n-i}} \operatorname{Ei}\left(-\frac{\alpha b}{a}-b\right) \\
&\left.+\frac{e^{-b}}{\alpha} \sum_{q=0}^{n+i-1} \sum_{j=0}^{n+i-q-1} \frac{j !(n+i)^{q}\left(\begin{array}{c}
n+i-q-1 \\
j
\end{array}\right) b^{n+i-q-j-1}}{\alpha^{q} a^{m-q}(\alpha / a+1)^{j+1}}\right] .
\end{aligned}
$$

$$
\begin{aligned}
\left\langle R_{l, k}\right\rangle=\log _{2} e \sum_{m=1}^{K(L-1)} \sum_{p=0}^{N-K} \frac{\prod_{n=1, n \neq m}^{K(L-1)}\left(1-\mu_{l, n} / \mu_{l, m}\right)^{-1}}{(N-K-p) !(-1)^{N-K-p} \mu_{l, m}}\left[-e^{\frac{1}{\beta_{l l k} p_{\mathrm{u}}}} \mathcal{I}_{0, N-K-p}\left(\frac{1}{\beta_{l l k}}, \frac{1}{\beta_{l l k} p_{\mathrm{u}}}, \frac{1}{\mu_{l, m}}-\frac{1}{\beta_{l l k}}\right)\right. \\
\left.\quad+\sum_{q=1}^{N-K-p} \frac{(q-1) !(-1)^{q}}{\beta_{l l k}^{N-K-p-q}} e^{\frac{1}{\mu_{l, m} p_{\mathrm{u}}}} \mu_{l, m}^{N+1-K-p-q} \Gamma\left(N+1-K-p-q, \frac{1}{\mu_{l, m} p_{\mathrm{u}}}\right)\right]
\end{aligned}
$$

\section{A. Uplink Rate Analysis}

From Proposition 1, the uplink ergodic rate from the $k$ th user in the $l$ th cell to its BS (in bits/s/Hz) is given by

$$
\left\langle R_{l, k}\right\rangle=\mathbb{E}_{X_{k}, Z_{l}}\left\{\log _{2}\left(1+\frac{p_{\mathrm{u}} X_{k}}{p_{\mathrm{u}} Z_{l}+1}\right)\right\} .
$$

By using (7) and (8), we can obtain the following analytical representation for the uplink ergodic rate [1]:

Proposition 2: The uplink ergodic rate from the $k$ th user in the $l$ th cell to its BS is given by (13) at the top at the page, where $U(\cdot, \cdot, \cdot)$ is the confluent hypergeometric function of the second kind [34, Eq. (9.210.2)], and $\mathcal{I}_{m, n}(a, b, \alpha)$ is given by (14), shown at the top of the page.

Proof: The proof can be found in [1, Section III-A].

In practice, users are located randomly within cells, such that large-scale fading coefficients for different users are different. This results in all diagonal elements of $\mathcal{A}_{l}$ being distinct. The following corollary corresponds to this practically important special case.

Corollary 1: If all diagonal elements of $\mathcal{A}_{l}$ are distinct, the ergodic rate in (13) reduces to (15), shown at the top of the page, with $\Gamma(a, x)=\int_{x}^{\infty} t^{a-1} e^{-t} d t$ being the upper incomplete gamma function [34, Eq. (8.350.2)].

Proof: For this case, substituting $\varrho\left(\mathcal{A}_{l}\right)=K(L-1)$, $\tau_{m}\left(\mathcal{A}_{l}\right)=1$, and

$$
\mathcal{X}_{m, 1}\left(\mathcal{A}_{l}\right)=\prod_{n=1, n \neq m}^{K(L-1)}\left(1-\frac{\mu_{l, n}}{\mu_{l, m}}\right)^{-1}
$$

into (13), and using the identity $U(1, a, x)=$ $e^{x} x^{1-a} \Gamma(a-1, x)$ [35, Eq. (07.33.03.0014.01)], we can obtain (15).

In addition to the exact result given by Proposition 2, we now derive an analytical lower bound on the ergodic achievable rate which is easier to evaluate:
Proposition 3: The uplink ergodic rate from the $k$ th user in the $l$ th cell to its BS is lower bounded by

$$
\begin{aligned}
& \left\langle R_{l, k}\right\rangle \geq \log _{2}\left(1+p_{\mathrm{u}} \beta_{l l k} \exp (\psi(N-K+1)\right. \\
& \left.\left.-p_{\mathrm{u}} \sum_{m=1}^{\varrho\left(\mathcal{A}_{l}\right)} \sum_{n=1}^{\tau_{m}\left(\mathcal{A}_{l}\right)} \mu_{l, m} n \mathcal{X}_{m, n}\left(\mathcal{A}_{l}\right)_{3} F_{1}\left(n+1,1,1 ; 2 ;-p_{\mathrm{u}} \mu_{l, m}\right)\right)\right)
\end{aligned}
$$

where $\psi(x)$ is Euler's digamma function [34, Eq. (8.360.1)], and ${ }_{p} F_{q}(\cdot)$ represents the generalized hypergeometric function with $p, q$ non-negative integers [34, Eq. (9.14.1)].

Proof: See Appendix A.

Remark 1: From (6), we have that

$$
\lim _{p_{\mathrm{u}} \rightarrow \infty} \gamma_{k} \stackrel{\mathrm{d}}{\sim} \frac{X_{k}}{\sum_{i \neq l}^{L}\left\|\boldsymbol{Y}_{i}\right\|^{2}} .
$$

The above result explicitly demonstrates that the SINR is bounded when $p_{\mathrm{u}}$ goes to infinity. This means that at high SNRs, we cannot improve the system performance by simply increasing the transmitted power of each user. The reason is that, when $p_{\mathrm{u}}$ increases, both the desired signal power and the interference power increase.

\section{B. SER Analysis}

In this section, we analyze the SER performance of the uplink for each user. Let $\mathcal{M}_{\gamma_{k}}(s)$ be the moment generating function (MGF) of $\gamma_{k}$. Then, using the well-known MGFbased approach [27], we can deduce the exact average SER of $M$-ary phase-shift keying ( $M$-PSK) as follows:

Proposition 4: The average SER of the uplink from the $k$ th user in the $l$ th cell to its BS for $M$-PSK is given by

$$
\operatorname{SER}_{k}=\frac{1}{\pi} \int_{0}^{\Theta} \mathcal{M}_{\gamma_{k}}\left(\frac{g_{\mathrm{MPSK}}}{\sin ^{2} \theta}\right) d \theta
$$


where $\Theta \triangleq \pi-\frac{\pi}{M}, g_{\mathrm{MPSK}} \triangleq \sin ^{2}(\pi / M)$, and

$$
\begin{aligned}
& \mathcal{M}_{\gamma_{k}}(s)=\sum_{m=1}^{\varrho\left(\mathcal{A}_{l}\right)} \sum_{n=1}^{\tau_{m}\left(\mathcal{A}_{l}\right)} \sum_{p=0}^{N-K+1}\left(\begin{array}{c}
N-K+1 \\
p
\end{array}\right) \mathcal{X}_{m, n}\left(\mathcal{A}_{l}\right) \\
& \times\left(\frac{-\beta_{l l k} s}{\beta_{l l k} s+1 / p_{\mathrm{u}}}\right)^{p}{ }_{2} F_{0}\left(n, p ;-; \frac{-\mu_{l, m}}{1 / p_{\mathrm{u}}+\beta_{l l k} s}\right) .
\end{aligned}
$$

Proof: See Appendix B.

It is also interesting to investigate the SER at high SNRs in order to obtain the diversity gain of the system under consideration. For this case $\left(p_{\mathrm{u}} \rightarrow \infty\right)$, by ignoring $1 / p_{\mathrm{u}}$ in (19), we obtain the asymptotic SER at high SNRs as

$$
\mathrm{SER}_{k}^{\infty}=\frac{1}{\pi} \int_{0}^{\Theta} \mathcal{M}_{\gamma_{k}}^{\infty}\left(\frac{g_{\mathrm{MPSK}}}{\sin ^{2} \theta}\right) d \theta
$$

where

$$
\begin{aligned}
\mathcal{M}_{\gamma_{k}}^{\infty}(s) & =\sum_{m=1}^{\varrho\left(\mathcal{A}_{l}\right)} \sum_{n=1}^{\tau_{m}\left(\mathcal{A}_{l}\right)} \sum_{p=0}^{N-K+1}\left(\begin{array}{c}
N-K+1 \\
p
\end{array}\right) \\
& \times \mathcal{X}_{m, n}\left(\mathcal{A}_{l}\right)(-1)^{p}{ }_{2} F_{0}\left(n, p ;-; \frac{-\mu_{l, m}}{\beta_{l l k} s}\right) .
\end{aligned}
$$

This implies that at high SNRs, the SER converges to a constant value that is independent of SNR; hence the diversity order, which is defined as $\lim _{p_{\mathrm{u}} \rightarrow \infty} \frac{-\log \operatorname{SER} R_{k}}{\log \left(p_{\mathrm{u}}\right)}$, is equal to zero. This phenomenon occurs due to the presence of interference. More precisely, as we can see from (17), when $p_{\mathrm{u}} \rightarrow \infty$, the SINR is bounded due to interference. The following corollary corresponds to the interesting case when all diagonal elements of $\mathcal{A}_{l}$ are distinct.

Corollary 2: If all diagonal elements of $\mathcal{A}_{l}$ are distinct, the exact and high-SNR MGF expressions in (19) and (21) reduce respectively to

$$
\begin{aligned}
\mathcal{M}_{\gamma_{k}}(s) & =\sum_{m=1}^{K(L-1)} \sum_{p=0}^{N-K+1}\left(\begin{array}{c}
N-K+1 \\
p
\end{array}\right) \mathcal{X}_{m, 1}\left(\mathcal{A}_{l}\right) \\
& \times \frac{\left(-\beta_{l l k} s\right)^{p} \mu_{l, m}^{-1}}{\left(1 / p_{\mathrm{u}}+\beta_{l l k} s\right)^{p-1}} e^{\frac{1 / p_{\mathrm{u}}+\beta_{l l k} s}{\mu_{l, m}}} \mathrm{E}_{p}\left(\frac{1 / p_{\mathrm{u}}+\beta_{l l k} s}{\mu_{l, m}}\right)
\end{aligned}
$$

$$
\begin{aligned}
\mathcal{M}_{\gamma_{k}}^{\infty}(s) & =\sum_{m=1}^{K(L-1)} \sum_{p=0}^{N-K+1}\left(\begin{array}{c}
N-K+1 \\
p
\end{array}\right) \mathcal{X}_{m, 1}\left(\mathcal{A}_{l}\right) \\
& \times \frac{(-1)^{p} \beta_{l l k} s}{\mu_{l, m}} e^{\frac{\beta_{l l k} s}{\mu_{l, m}}} \mathrm{E}_{p}\left(\frac{\beta_{l l k} s}{\mu_{l, m}}\right)
\end{aligned}
$$

where $\mathrm{E}_{n}(z)=\int_{1}^{\infty} t^{-n} e^{-z t} d t, n=0,1,2, \ldots, \operatorname{Re}(z)>0$, is the exponential integral function of order $n$ [35, Eq. (06.34.02.0001.01)].

Proof: Following a similar methodology as in Corollary 1 and using the identity

$$
{ }_{2} F_{0}(1, p ;-;-x)=\frac{1}{x} e^{1 / x} \mathrm{E}_{p}\left(\frac{1}{x}\right)
$$

we arrive at the desired results (22) and (23). Note that (24) is obtained by using [38, Eq. (8.4.51.1)], [38, Eq. (8.2.2.15)], [38, Eq. (8.4.16.14)] and [39, Eq. (46)].
From (18), we can see that to compute the SER we have to perform a finite integration over $\theta$. To avoid this integration, we can apply the tight approximation of [36] on (18), to get

$$
\begin{aligned}
\mathrm{SER}_{k} & \approx\left(\frac{\Theta}{2 \pi}-\frac{1}{6}\right) \mathcal{M}_{\gamma_{k}}\left(g_{\mathrm{MPSK}}\right)+\frac{1}{4} \mathcal{M}_{\gamma_{k}}\left(\frac{4 g_{\mathrm{MPSK}}}{3}\right) \\
& +\left(\frac{\Theta}{2 \pi}-\frac{1}{4}\right) \mathcal{M}_{\gamma_{k}}\left(\frac{g_{\mathrm{MPSK}}}{\sin ^{2} \Theta}\right) .
\end{aligned}
$$

The above expression is easier to evaluate compared to (18).

\section{Outage Probability Analysis}

The main goal of this section is to analytically assess the outage probability of multicell MU-SIMO systems with ZF processing at the BS. Especially for the case of non-ergodic channels (e.g. quasi-static or block-fading channels), it is appropriate to resort to the notion of outage probability to characterize the system performance. The outage probability, $P_{\text {out }}$, is defined as the probability that the instantaneous SINR, $\gamma_{k}$, falls below a given threshold value $\gamma_{\text {th }}$, i.e.,

$$
P_{\text {out }} \triangleq \operatorname{Pr}\left(\gamma_{k} \leq \gamma_{\text {th }}\right)
$$

With this definition in hand, we can present the following novel, exact result:

Proposition 5: The outage probability of transmission from the $k$ th user in the $l$ th cell to its $\mathrm{BS}$ is given by

$$
\begin{aligned}
P_{\text {out }} & =1-\exp \left(-\frac{\gamma_{\text {th }}}{p_{\mathrm{u}} \beta_{l l k}}\right) \sum_{m=1}^{\varrho\left(\mathcal{A}_{l}\right)} \sum_{n=1}^{\tau_{m}\left(\mathcal{A}_{l}\right)} \sum_{p=0}^{N-K} \sum_{q=0}^{p}\left(\begin{array}{l}
p \\
q
\end{array}\right) \frac{\left(\frac{\gamma_{\text {th }}}{\beta_{l l k}}\right)^{p}}{p !} \\
& \times \mathcal{X}_{m, n}\left(\mathcal{A}_{l}\right) \frac{\mu_{l, m}^{-n}}{(n-1) !} \frac{\Gamma(n+q) p_{\mathrm{u}}^{q-p}}{\left(1 / \mu_{l, m}+\gamma_{\mathrm{th}} / \beta_{l l k}\right)^{n+q}}
\end{aligned}
$$

Proof: See Appendix C.

Note that the exponential integral function and confluent hypergeometric functions appearing in Propositions 2, 4, and 5 are built-in functions and can be easily evaluated by standard mathematical software packages, such as MATHEMATICA or MATLAB. We now recall that we are typically interested in small outage probabilities (e.g., in the order of 0.01, 0.001 etc). In this light, when $\gamma_{\text {th }} \rightarrow 0$, we can obtain the following asymptotic result:

$$
\begin{aligned}
P_{\text {out }}^{\infty} & =1-\sum_{m=1}^{\varrho\left(\mathcal{A}_{l}\right)} \sum_{n=1}^{\tau_{m}\left(\mathcal{A}_{l}\right)} \sum_{p=0}^{N-K} \frac{\left(\frac{\gamma_{\text {th }}}{\beta_{l l k}}\right)^{p}}{p !} \mathcal{X}_{m, n}\left(\mathcal{A}_{l}\right) \\
& \times \frac{\mu_{l, m}^{-n}}{(n-1) !} \frac{\Gamma(n+p)}{\left(1 / \mu_{l, m}+\gamma_{\text {th }} / \beta_{l l k}\right)^{n+p}} .
\end{aligned}
$$

The above result is obtained by keeping the dominant term $p=q$ in (27) and letting $\gamma_{\text {th }} \rightarrow 0$. Similarly to the SER case, $P_{\text {out }}^{\infty}$ is independent of the SNR, thereby reflecting the deleterious impact of interference. Furthermore, for the case described in Corollaries 1 and 2, we can get the following simplified results:

Corollary 3: If all diagonal elements of $\mathcal{A}_{l}$ are distinct, the exact and high-SNR outage probability expressions in (27) and 
(28) reduce respectively to

$$
\begin{aligned}
P_{\text {out }} & =1-\exp \left(-\frac{\gamma_{\text {th }}}{p_{\mathrm{u}} \beta_{l l k}}\right) \sum_{m=1}^{K(L-1)} \sum_{p=0}^{N-K} \sum_{q=0}^{p} \frac{\left(\frac{\gamma_{\text {th }}}{\beta_{l l k}}\right)^{p}}{(p-q) !} \\
& \times \frac{\mathcal{X}_{m, 1}\left(\mathcal{A}_{l}\right)}{\mu_{l, m}} \frac{p_{\mathrm{u}}^{q-p}}{\left(1 / \mu_{l, m}+\gamma_{\mathrm{th}} / \beta_{l l k}\right)^{q+1}} \\
P_{\text {out }}^{\infty} & =1-\sum_{m=1}^{K(L-1)} \sum_{p=0}^{N-K}\left(\frac{\gamma_{\text {th }}}{\beta_{l l k}}\right)^{p} \frac{\mathcal{X}_{m, 1}\left(\mathcal{A}_{l}\right) / \mu_{l, m}}{\left(\frac{1}{\mu_{l, m}}+\frac{\gamma_{\text {th }}}{\beta_{l l k}}\right)^{1+p}} .
\end{aligned}
$$

\section{Asymptotic $(N \rightarrow \infty)$ Analysis}

As discussed in Remark 1, we cannot improve the multicell MU-SIMO system performance by simply increasing the transmit power. However, we can improve the system performance by using a large number of BS antennas. Due to the array gain and diversity effects, when $N$ increases, the received powers of both the desired and interference signals increase. Yet, based on the asymptotic orthogonal property of the channel vectors between the users and the BS, when $N$ is large, interference can be significantly reduced even with a simple ZF receiver [20], [21]. In this section, we analyze the asymptotic performance for large $N$. We assume that when $N$ increases, the elements of the channel matrix are still independent. To guarantee the independence of the channels, the antennas have to be sufficiently well separated. Note that the physical size of the antenna array can be small even with very large $N$. For example, at $2.6 \mathrm{GHz}$, a cylindrical array with 128 antennas, which comprises 4 circles of 16 dual polarized antenna elements (distance between adjacent antennas is about $6 \mathrm{~cm}$ which is half a wavelength), occupies only a physical size of $28 \mathrm{~cm} \times 29 \mathrm{~cm} \mathrm{[28].}$

1) Fixed $p_{\mathrm{u}}, K$, and $N \rightarrow \infty$ : Intuitively, when the number of BS antennas $N$ grows large, the random vectors between the BS and the users as well as the noise vector at the BS become pairwisely orthogonal and hence, interference from users in other cells can be canceled out. At the same time, due to the array gain effect, the impact of thermal noise is minimized too. This intuition is confirmed by the following analysis. Since $X_{k}$ has an Erlang distribution with shape parameter $N-K+1$ and scale parameter $\beta_{l l k}, X_{k}$ can be represented as

$$
X_{k}=\frac{\beta_{l l k}}{2} \sum_{i=1}^{2(N-K+1)} Z_{i}^{2}
$$

where $Z_{1}, Z_{2}, \ldots, Z_{2(N-K+1)}$ are independent, standard normal RVs. Substituting (31) into (9), and dividing the denominator and the numerator of $\gamma_{k}$ by $2(N-K+1)$, as $N \rightarrow \infty$, we obtain

$$
\gamma_{k}=\frac{p_{\mathrm{u}} \frac{\beta_{l l k}}{2} \sum_{i=1}^{2(N-K+1)} Z_{i}^{2} /(2(N-K+1))}{\left(p_{\mathrm{u}} \sum_{i \neq l}^{L}\left\|\boldsymbol{Y}_{i}\right\|^{2}+1\right) /(2(N-K+1))} \stackrel{\text { a.s. }}{\rightarrow} \infty
$$

where (32) is obtained by using the law of large numbers, i.e., the numerator converges to $p_{\mathrm{u}} \beta_{l l k} / 2$, while the denominator converges to 0 . The above result reveals that, when the number of BS antennas goes to infinity, the effects of interference and noise disappear. Therefore, by increasing $N$, the SINR grows without limit. Similar conclusions were presented in [20].
2) Fixed $p_{\mathrm{u}}, \kappa=N / K$, and $N \rightarrow \infty$ : This is an interesting asymptotic scenario since in practice, the number of BS antennas, $N$, is large but may not be much greater than the number of users $K$. For this case, the property stating that the channel vectors between users and the BS are pairwisely orthogonal when $N \rightarrow \infty$ is not valid. In other words, $\boldsymbol{H}_{l i}^{H} \boldsymbol{H}_{l i}$ does not converge point-wisely to an "infinite-size identity matrix" [22]. Thus, intercell interference cannot be canceled out. Since $\boldsymbol{Y}_{i} \sim \mathcal{C N}\left(\mathbf{0}, \boldsymbol{D}_{l i}\right)$, it can be represented as

$$
\boldsymbol{Y}_{i}=\boldsymbol{w}_{i}^{H} \boldsymbol{D}_{l i}^{1 / 2}
$$

where $\boldsymbol{w}_{i} \sim \mathcal{C N}\left(\mathbf{0}, \boldsymbol{I}_{K}\right)$. From (9), (31), and (33), $\gamma_{k}$ can be expressed as

$$
\gamma_{k}=\frac{p_{\mathrm{u}} \frac{\beta_{l l k}}{2} \sum_{i=1}^{2(N-K+1)} Z_{i}^{2}}{p_{\mathrm{u}} \sum_{i \neq l}^{L} \boldsymbol{w}_{i}^{H} \boldsymbol{D}_{l i} \boldsymbol{w}_{i}+1} .
$$

By dividing the numerator and denominator of $\gamma_{k}$ in (34) by $2(N-K+1)$, we obtain

$$
\gamma_{k}=\frac{p_{\mathrm{u}} \frac{\beta_{l l k}}{2} \sum_{i=1}^{2(N-K+1)} Z_{i}^{2} /(2(N-K+1))}{\left(p_{\mathrm{u}} \sum_{i \neq l}^{L} \boldsymbol{w}_{i}^{H} \boldsymbol{D}_{l i} \boldsymbol{w}_{i}+1\right) /(2(N-K+1))} .
$$

Since $N / K=\kappa$, (35) can be rewritten as

$$
\gamma_{k}=\frac{p_{\mathrm{u}} \beta_{l l k}\left(\kappa-1+\frac{1}{K}\right) \sum_{i=1}^{2(N-K+1)} Z_{i}^{2} /(2(N-K+1))}{\left(p_{\mathrm{u}} \sum_{i \neq l}^{L} \boldsymbol{w}_{i}^{H} \boldsymbol{D}_{l i} \boldsymbol{w}_{i}+1\right) / K} .
$$

From (36), by using the law of large numbers and the trace lemma from [29, Lemma 13], i.e., ${ }^{4}$

$$
\frac{1}{K} \boldsymbol{w}_{i}^{H} \boldsymbol{D}_{l i} \boldsymbol{w}_{i}-\frac{1}{K} \operatorname{Tr}\left(\boldsymbol{D}_{l i}\right) \stackrel{\text { a.s. }}{\rightarrow} 0, \text { as } K \rightarrow \infty
$$

we obtain

$$
\gamma_{k}-\frac{\beta_{l l k}(\kappa-1)}{\sum_{i=1, i \neq l}^{L} \frac{1}{K} \operatorname{Tr}\left(\boldsymbol{D}_{l i}\right)} \stackrel{\text { a.s. }}{\rightarrow} 0, \text { as } N \rightarrow \infty, \text { and } N / K=\kappa .
$$

Therefore a deterministic approximation, $\bar{\gamma}_{k}$, of $\gamma_{k}$ is given by

$$
\bar{\gamma}_{k}=\frac{\beta_{l l k}(\kappa-1)}{\sum_{i=1, i \neq l}^{L} \frac{1}{K} \operatorname{Tr}\left(\boldsymbol{D}_{l i}\right)} .
$$

It is interesting to note that the signal-to-interference ratio (SIR) expression (38) is independent of the transmit power, and increases monotonically with $\kappa$. Therefore, for an arbitrarily small transmit power, the SIR (38) can be approached arbitrarily closely by using a sufficiently large number of antennas and users. The reason is that since the number of users $K$ is large, the system is interference-limited, so if every user reduces its power by the same factor then the limiting SIR is unchanged. Furthermore, from (38), when $\kappa \rightarrow \infty$ (this is equivalent to the case $N \gg K$ ), the $\operatorname{SIR} \bar{\gamma}_{k} \rightarrow \infty$, as $N \rightarrow \infty$, which is consistent with (32).

\footnotetext{
${ }^{4}$ Note that the trace lemma holds if $\lim \sup _{K} \mathbb{E}\left\{\frac{1}{K} \operatorname{Tr}\left(\boldsymbol{D}_{l i} \boldsymbol{D}_{l i}^{H}\right)^{2}\right\}<\infty$ which is equivalent to $\mathbb{E}\left\{\beta_{\text {lik }}^{4}\right\}<\infty$ [29, Remark 3]. For example, if $\beta_{l i k}$ is a lognormal RV with standard deviation of $\sigma$, then $\mathbb{E}\left\{\beta_{l i k}^{4}\right\}=e^{8 \sigma^{2}}[30]$. Evidently, for the vast majority of practical cases of interest, the standard deviation is finite, which makes the fourth moment bounded.
} 


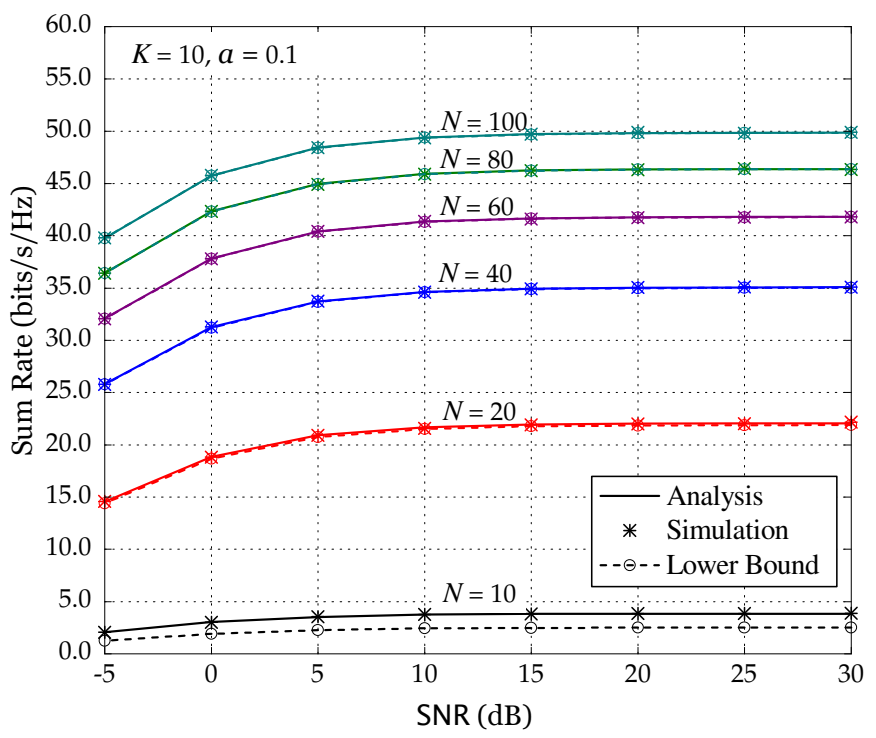

Fig. 1. Simulated uplink sum rate, analytical expression and lower bound versus the SNR ( $L=4, K=10$, and $a=0.1)$.

3) Fixed $N p_{\mathrm{u}}, N \rightarrow \infty$ : Let $p_{\mathrm{u}}=E_{\mathrm{u}} / N$, where $E_{\mathrm{u}}$ is fixed. From (32), we have

$$
\gamma_{k}=\frac{E_{\mathrm{u}} \frac{\beta_{l l k}}{2} \frac{\sum_{i=1}^{2(N-K+1)} Z_{i}^{2}}{2(N-K+1)} \frac{2(N-K+1)}{N}}{\frac{E_{\mathrm{u}}}{N} \sum_{i \neq l}^{L}\left\|\boldsymbol{Y}_{i}\right\|^{2}+1} .
$$

Then, again using the law of large numbers and the trace lemma, we obtain

$$
\begin{aligned}
& \gamma_{k}-\beta_{l l k} E_{\mathrm{u}} \stackrel{\text { a.s. }}{\rightarrow} 0, \text { as } N \rightarrow \infty, \text { and fixed } K \\
& \gamma_{k}-\frac{\beta_{l l k} E_{\mathrm{u}}(1-1 / \kappa)}{\frac{E_{\mathrm{u}}}{\kappa} \sum_{i \neq l}^{L} \frac{1}{K} \operatorname{Tr}\left(\boldsymbol{D}_{l i}\right)+1} \stackrel{\text { a.s. }}{\rightarrow} 0, \text { as } N \rightarrow \infty, \frac{N}{K}=\kappa .
\end{aligned}
$$

These results show that by using a very large antenna array at the BS, we can cut the transmit power at each user proportionally to $1 / N$ while maintaining a desired quality-ofservice. This result was originally established in [20] for the case when $N \gg K \gg 1$ whereas herein, we have generalized this result to the regime where $N \gg 1$. Again, we can see that, when $\kappa$ tends to infinity, the two asymptotic results (40) and (41) coincide.

Remark 2: We can see from (40) that when $N \rightarrow \infty$ and $K$ is fixed, the effects of interference and small-scale fading disappear. The only remaining effect is noise. Let us define the "massive MIMO effect" as the case where the system is ultimately limited by noise. ${ }^{5}$ From (41), when $N$ grows large while keeping a finite $\kappa$, the system is still limited by interference from other cells. This interference depends mainly on $\kappa$ (the degrees of freedom), and when $\kappa \rightarrow \infty$, we operate under massive MIMO conditions. Therefore, an interesting question is: How many degrees of freedom $\kappa$ are

\footnotetext{
${ }^{5}$ The term "massive MIMO effect" was also used in [23] but in a different meaning, namely referring to the case when the system performance is limited by pilot contamination, due to the use of non-orthogonal pilots in different cells for the uplink training phase. However, here we assume orthogonal pilot sequences in different cells, and we consider a particular operating condition where the transmit power is very small $\left(p_{\mathrm{u}} \sim 1 / N\right)$.
}

needed in order to make interference small compared to noise (i.e., to reach the massive MIMO condition)? Mathematically speaking, we seek to find $\kappa$ that satisfies

$$
\log _{2}\left(1+\frac{\beta_{l l k} E_{\mathrm{u}}(1-1 / \kappa)}{E_{\mathrm{u}} / \kappa \sum_{i=1, i \neq l}^{L} \frac{1}{K} \operatorname{Tr}\left(\boldsymbol{D}_{l i}\right)+1}\right) \geq \eta R_{k, \infty}
$$

for a desired $\eta \in(0,1)$, where $R_{k, \infty} \triangleq \log _{2}\left(1+\beta_{l l k} E_{\mathrm{u}}\right)$ is the ultimate rate which corresponds to the regime where $N \gg K \gg 1$. We, more closely, address this fundamental issue via simulations in Section V.

Remark 3: When $N \gg K \gg 1$ and $p_{\mathrm{u}}=E_{\mathrm{u}} / N$, using the property $\psi(x)=\ln (x)+1 / x+\mathcal{O}\left(1 / x^{2}\right)$, and observing that the second term of the exponential function approaches zero, we can simplify (16) to get $\left\langle R_{l, k}\right\rangle \geq \log _{2}\left(1+\beta_{l l k} E_{\mathrm{u}}\right)$, which coincides with (40) for fixed $K$. This implies that the proposed lower bound becomes exact at large $N$.

\section{Numerical Results}

In this section, we provide some numerical results to verify our analysis. Firstly, we consider a simple scenario where the large-scale fading is fixed. This setting enables us to validate the accuracy of our proposed analytical expressions as well as study the fundamental effects of intercell interference, number of BS antennas, transmit power of each user on the system performance. We then consider a more practical scenario that accounts for random user locations and incorporates smallscale fading as well as large-scale fading including path-loss and lognormal shadow fading.

\section{A. Scenario I}

We consider a multicell system with 4 cells sharing the same frequency band. ${ }^{6}$ In all examples, except Fig. 4 , we choose $K=10$. We assume that $\beta_{l l k}=1$ and $\beta_{l j k}=a, \forall j \neq l$, $k=1,2, \ldots, K$. Since $a$ represents the effect of interference from other cells, it can be regarded as an intercell interference factor. Furthermore, we define SNR $\triangleq p_{\mathrm{u}}$.

Figure 1 shows the uplink sum rate per cell versus SNR, at intercell interference factor $a=0.1$ and for $N=10$, 20, 40, 60, 80 and 100. The simulation curves are obtained by performing Monte-Carlo simulations using (5), while the analytical and bound curves are computed via (13) and (16), respectively. As expected, when $N$ increases, the sum rate increases too. However, at high SNRs, the sum rate converges to a deterministic constant which verifies our analysis (17). Furthermore, a larger value of $N$ makes the bound tighter. This is due to the fact that when $N$ grows large, things that were random before become deterministic and, hence, Jensen's inequality used in (44) will hold with equality (see Remark 3). Therefore, the bound can very efficiently approximate the rate when $N$ is large. It can be also seen that, even for moderate number of antennas $(N \gtrsim 20)$, the bound becomes almost exact across the entire SNR range.

\footnotetext{
${ }^{6}$ This is a circular variant of the linear Wyner model with 4 cells. This classical model can efficiently capture the fundamental structure of a cellular network and can facilitate the performance analysis [11], [40].
} 
This article has been accepted for publication in a future issue of this journal, but has not been fully edited. Content may change prior to final publication.

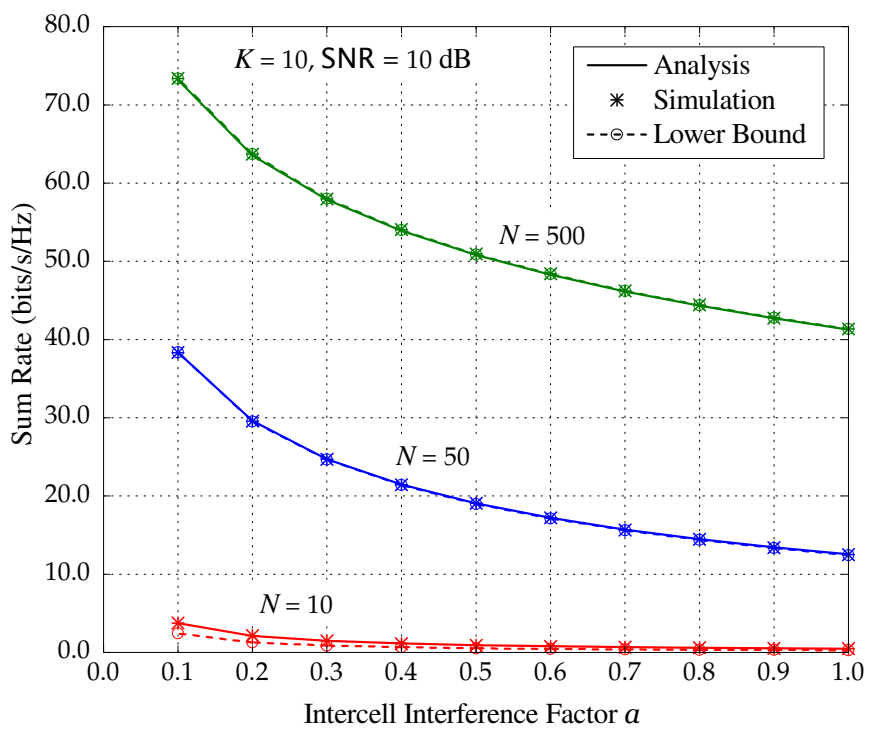

Fig. 2. Simulated sum rate, analytical expression and lower bound versus the intercell interference factor $a(L=4, K=10$, and SNR $=10 \mathrm{~dB})$.

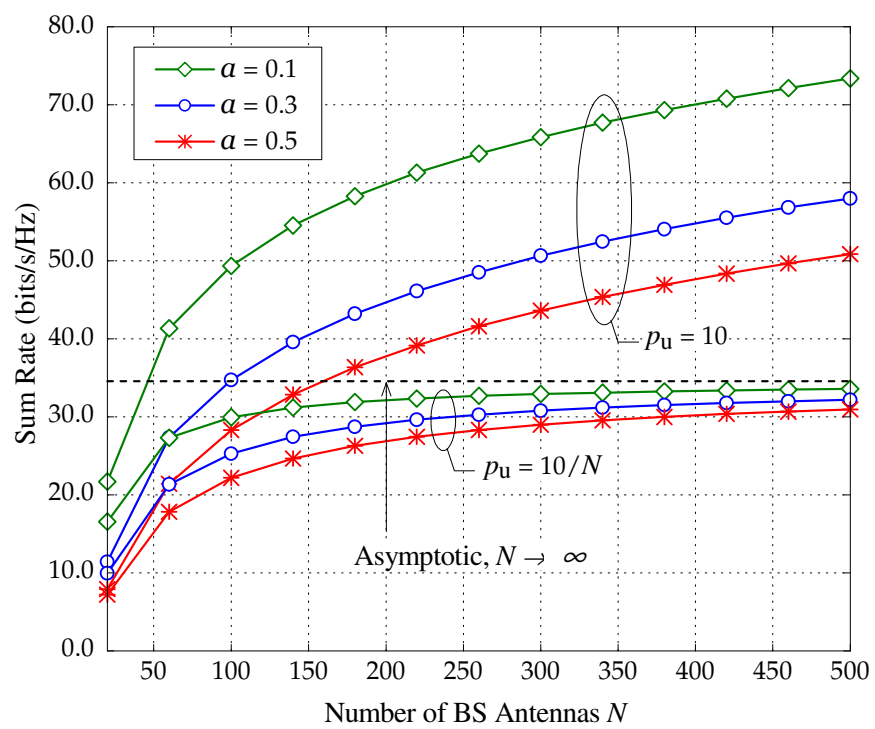

Fig. 3. Analytical uplink sum rate versus the number of BS antennas $N$ $(L=4, K=10, a=0.1,0.3$, and 0.5$)$.

The effect of interference for different $N$ is shown in Fig. 2. Again, the simulated and analytical results match exactly, and the bound is very tight. Interestingly, its tightness does not depend on the interference level but on the number of BS antennas. When the intercell interference factor increases (and, hence, interference increases), the sum rate decreases significantly. On the other hand, the effect of interference decreases when $N$ grows large. For example, at $a=0.1$, the sum rates are $3.76,38.35$, and 73.20 for $N=10,50$, and 500, respectively, while at $a=0.5$, the sum rates are respectively $0.93,19.10$, and 50.80 for $N=10,50$, and 500 . This means that when increasing intercell interference factor from 0.1 to 0.5 , the sum rates are reduced by $75.27 \%, 50.20 \%$, and $30.60 \%$ for $N=10,50$, and 500 , respectively.

The power efficiency of large array systems is investigated in Fig. 3. Figure 3 shows the uplink sum rate per cell versus

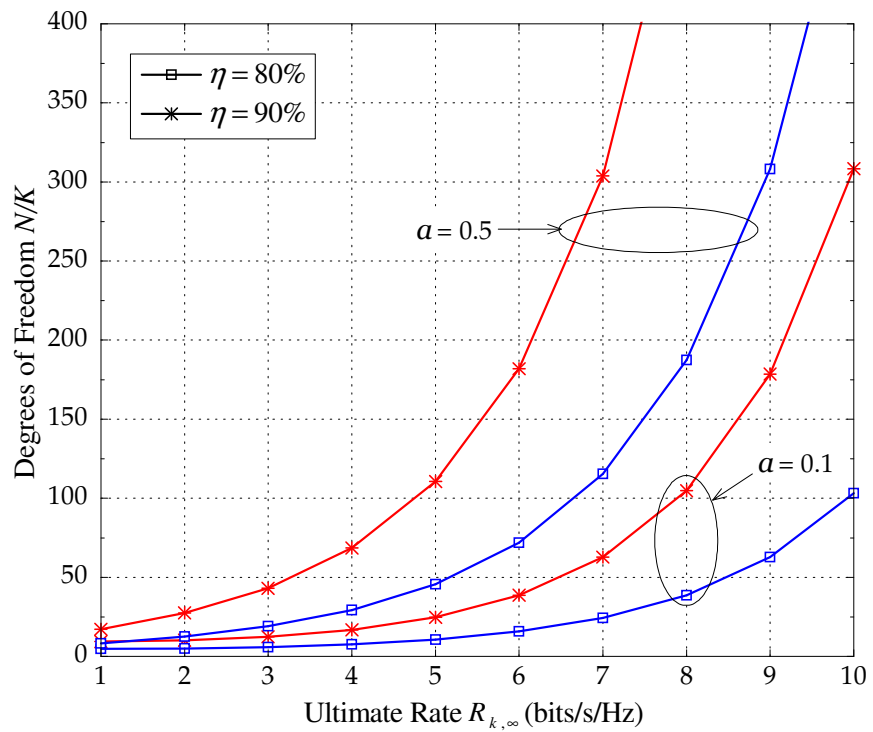

Fig. 4. Degrees of freedom $\kappa$ required to achieve $\eta R_{k, \infty}$ versus $R_{k, \infty}$ $(L=4, a=0.1$ and 0.5$)$.

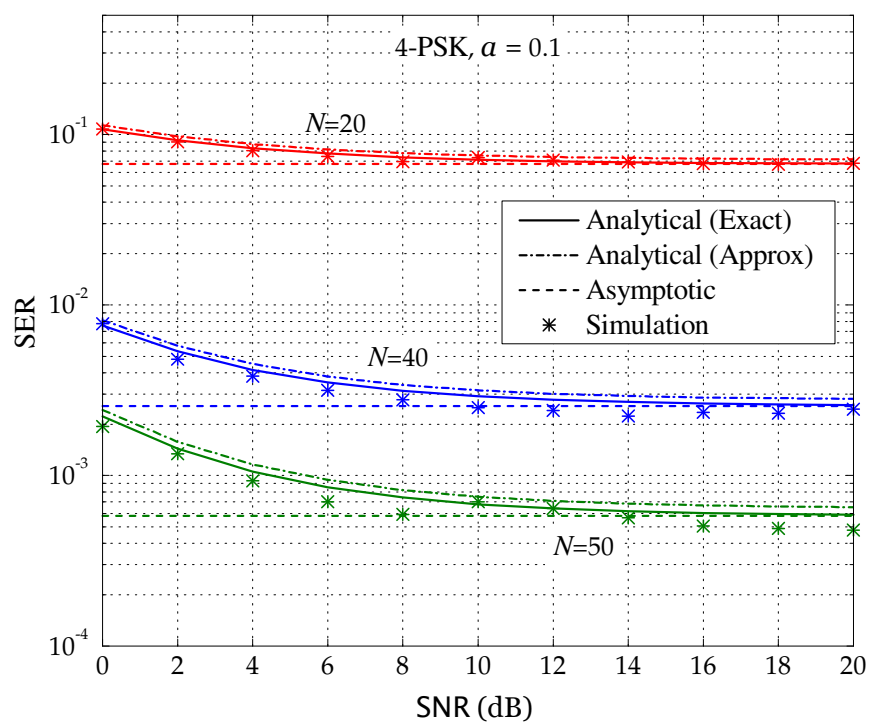

Fig. 5. Simulated average SER and analytical expression versus the SNR for 4-PSK $(L=4, K=10$ and $a=0.1)$.

$N$ at $a=0.1,0.3$, and 0.5 for the cases of $p_{\mathrm{u}}=10$ and $p_{\mathrm{u}}=10 / N$. As expected, with $p_{\mathrm{u}}=10 / N$, the sum rate converges to a constant value when $N$ increases regardless of the effects of interference, and with $p_{\mathrm{u}}=10$, the sum rate grows without bound (logarithmically fast with $N$ ) when $N$ increases (see (32) and (40)).

Figure 4 shows the required number of degrees of freedom $\kappa$ to achieve $80 \%(\eta=0.8)$ and $90 \%(\eta=0.9)$ of a given ultimate rate $R_{k, \infty}$, for $a=0.1$, and $a=0.5$. We use (42) to determine $\kappa$. We can see that $\kappa$ increases with $R_{k, \infty}$. Therefore, for multicell systems, the BS can serve more users with low data rates. This is due to the fact that when $R_{k, \infty}$ increases, the transmit power increases and, hence, interference also increases. Then, we need more degrees of freedom to mitigate interference. For the same reason, we 


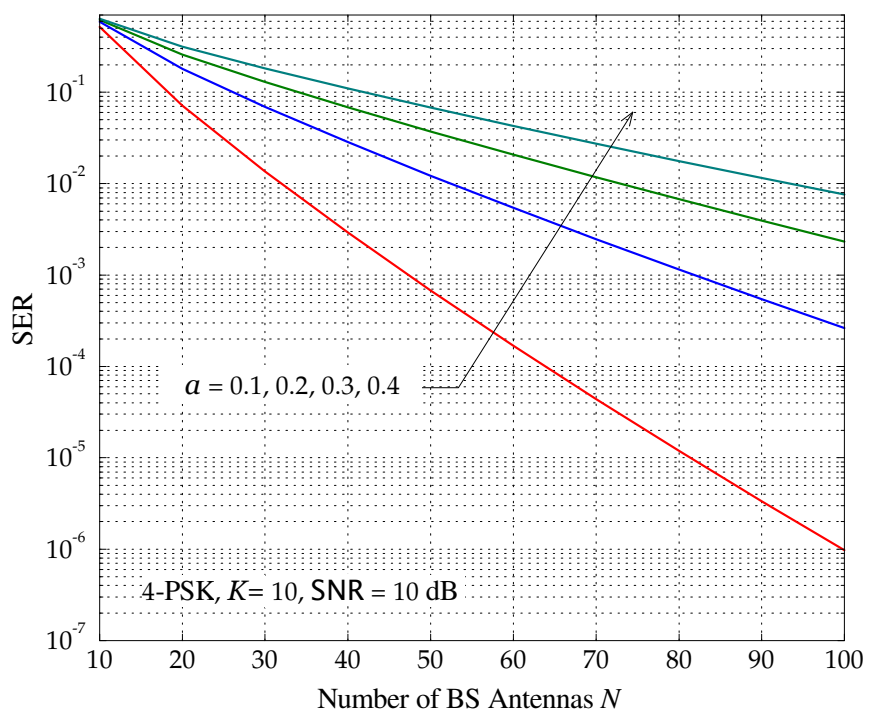

Fig. 6. Analytical average SER versus the number of BS antennas $N(L=4$, $K=10, a=0.1,0.2,0.3$, and 0.4 ).

can observe that when the interference factor $a$ increases, the required $\kappa$ increases as well.

In Fig. 5, the analytical SER curves are compared with the outputs of a Monte-Carlo simulation for different $N$. Here, we choose 4-PSK and $a=0.1$. The "Analytical (Exact)" curves are computed using Proposition 4, and the "Analytical (Approx)" curves are generated using (25). The high-SNR curves, generated via (20), are also overlaid. It can be easily observed that the analytical results coincide with the simulation results. Furthermore, we can see that the "Analytical (Approx)" curves are accurate in all cases. As in the analysis of the sum rate, when the SNR is moderately large, the SER decreases very slowly and approaches an error floor (the asymptotic SER) due to interference, when SNR grows large. Yet, we can improve the system performance by increasing the number of BS antennas. The advantages of using large antenna arrays on the SER can be further verified in Fig. 6, where the SER is plotted as a function of $N$ for different intercell interference factors and 4-PSK, at SNR $=10 \mathrm{~dB}$. We can see that the system performance improves systematically when we increase $N$.

\section{B. Scenario II}

We consider a hexagonal cellular network where each cell has a radius (from center to vertex) of $1000 \mathrm{~m}$. In each cell, $K=10$ users are located uniformly at random and we assume that no user is closer to the BS than $r_{\mathrm{h}}=100 \mathrm{~m}$. The largescale fading is modeled via $\beta_{l i k}=z_{l i k} /\left(r_{l i k} / r_{\mathrm{h}}\right)^{\nu}$, where $z_{l i k}$ represents a lognormal RV with standard deviation of 8 $\mathrm{dB}, r_{l i k}$ is the distance between the $k$ th user in the $i$ th cell to the $l$ th BS, and $\nu$ is the path loss exponent. We choose $\nu=3.8$ for our simulations. Furthermore, we assume that the transmitted data is modulated using OFDM. Let $T_{\mathrm{s}}$ and $T_{\mathrm{u}}$ be the OFDM symbol duration and useful symbol duration, respectively. Then, we define the net uplink rate of the $k$ th

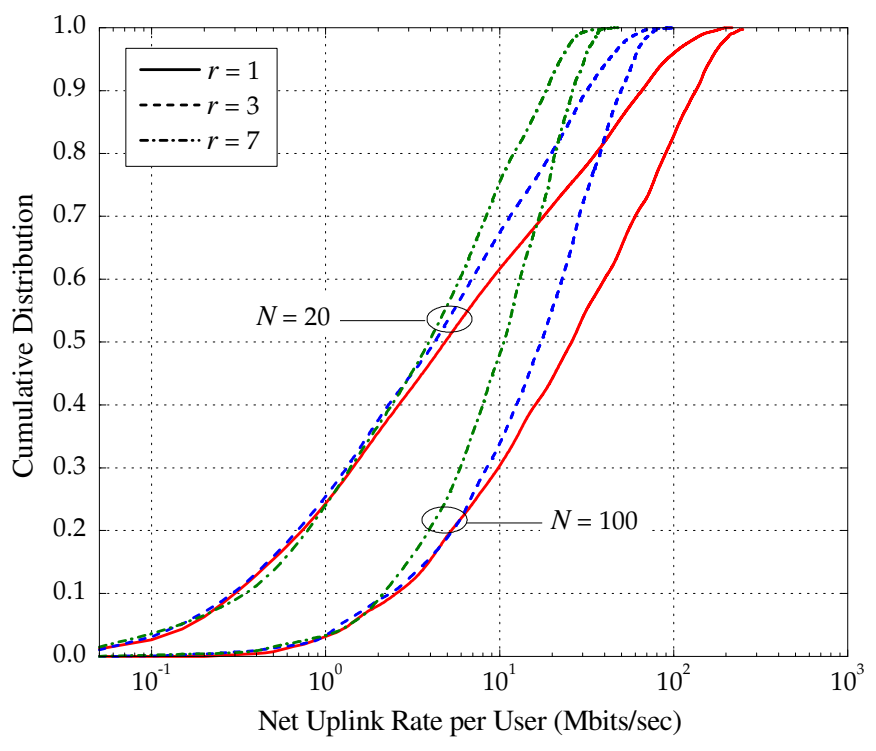

Fig. 7. Cumulative distribution of the net uplink rate per user for frequencyreuse factors 1,3 , and $7\left(N=20,100, \mathrm{SNR}=10 \mathrm{~dB}, \sigma_{\text {shadow }}=8 \mathrm{~dB}\right.$, and $\nu=3.8)$.

user in the $l$ th cell as follows [21]:

$$
R_{l, k}^{\text {net }}=\frac{B}{r} \frac{T_{\mathrm{u}}}{T_{\mathrm{s}}} \log _{2}\left(1+\frac{p_{\mathrm{u}} X_{k}}{p_{\mathrm{u}} Z_{l}+1 / r}\right)
$$

where $B$ is the total bandwidth, and $r$ is the frequencyreuse factor. Note that (43) is obtained by using the result in Proposition 1. For our simulations, we choose parameters that resemble those of the LTE standard [21]: $T_{\mathrm{s}}=71.4 \mu \mathrm{sec}$, and $T_{\mathrm{u}}=66.7 \mu \mathrm{sec}$. We further assume that $B=20 \mathrm{MHz}$. We neglect the effects of all users in all cells which are outside a circular region with a radius (from the $l$ th BS) of 8000 meters. This is reasonable since the interference from all users which are outside this region is negligible due to very high path loss.

Figure 7 shows the cumulative distribution of the net uplink rate per user for different frequency-reuse factors $r=1,3$, and 7 , and different number of BS antennas $N=20,100$. We can see that the number of BS antennas has a very strong impact on the performance. The probability that the net uplink rate is smaller than a given indicated rate decreases significantly when $N$ increases. We consider the $95 \%$-likely rates, i.e., the rate is greater than or equal to this indicated rate with probability 0.95 . We can see that the 95\%-likely rates increase with $N$; for example, with frequency-reuse factor of 1 , increasing the number of BS antennas from 20 to 100 yields a 8 -fold improvement in the $95 \%$-likely rate (from 0.170 Mbits/sec to $1.375 \mathrm{Mbits} / \mathrm{sec}$ ). Furthermore, when $N$ is large, the random channel becomes deterministic and hence, the probability that the uplink rate is around its mean becomes inherently higher.

When comparing the effects of using frequency-reuse factors, we can see that, at high rates (and hence at high SNR), smaller reuse factors are preferable, and vice versa at low rates. The reason is that, the rate in (43) is affected by the reuse factor through the pre-log factor and the SINR term. When the reuse factor increases, the pre-log factor decreases, while the SINR increases. At high SNRs, the pre-log factor has larger 
This article has been accepted for publication in a future issue of this journal, but has not been fully edited. Content may change prior to final publication.

TABLE I

UPLINK PERFORMANCE OF ZF RECEIVERS WITH FREQUENCY-REUSE FACTORS 1,3 , AND 7 , FOR $p_{\mathrm{u}}=10 \mathrm{~dB}, \sigma_{\text {shadow }}=8 \mathrm{~dB}$, AND $\nu=3.8$

\begin{tabular}{|c|c|c|c|c||}
\hline $\begin{array}{c}\text { Frequency } \\
\text { Reuse } \\
\text { Factor }\end{array}$ & \multicolumn{2}{|c|}{$\begin{array}{c}0.95 \text {-likely Net } \\
\text { Uplink Rate per } \\
\text { User (Mbits/sec) }\end{array}$} & \multicolumn{2}{c|}{$\begin{array}{c}\text { Mean of the Net } \\
\text { Uplink Rate per } \\
\text { User (Mbits/sec) }\end{array}$} \\
\cline { 2 - 5 } & $N=20$ & $N=100$ & $N=20$ & $N=100$ \\
\hline \hline 1 & 0.170 & 1.375 & 20.295 & 48.380 \\
\hline 3 & 0.150 & 1.205 & 10.668 & 21.945 \\
\hline 7 & 0.145 & 1.350 & 6.731 & 12.546 \\
\hline
\end{tabular}

impact on the rate and vice versa at low SNRs. Furthermore, we can observe that the gap between the performance of different reuse factors becomes larger when $N$ increases. This is due to the fact that, when $N$ is large, the intercell interference can be notably reduced; as a consequence, the bandwidth used has a larger impact on the system performance. Table I summarizes the $95 \%$-likely net uplink rates as well as their mean values.

\section{CONCLUSION}

In this paper, we analyzed in detail the uplink performance of data transmission from $K$ single-antenna users in one cell to its $N$-antenna BS in the presence of interference from other cells. The BS uses ZF to detect the transmitted signals. We derived exact analytical expressions for the most important figures of merit, namely the uplink rate, SER, and outage probability, assuming that the channel between the users and the BS is affected by Rayleigh fading, lognormal shadow fading, and path loss.

Theoretically, when $N$ increases we obtain array and diversity gains, which affect both the desired and interference signals. Hence, from this perspective the performance is not dramatically affected. However, when $N$ is large, the channel vectors between the users and the BS are pairwisely asymptotically orthogonal and, hence, interference can be canceled out with a simple linear $\mathrm{ZF}$ receiver (for fixed number of users). In the case that the ratio between the number of BS antennas and the number of users is fixed, the intercell interference persists when the number of antennas grows large, but we can still obtain an array gain. As a consequence, by using a large antenna array, the performance of the multicell system improves significantly. Furthermore, we investigated the achievable power efficiency when using large antenna arrays at the BSs. Large antenna arrays enable us to reduce the transmitted power of each user proportionally to $1 / N$ with no performance degradation, provided that the BS has perfect CSI of all users in its cell. We further elaborated on the massive MIMO effect and the impact of frequency-reuse factors.

\section{APPENDIX}

\section{A. Proof of Proposition 3}

From (12), the uplink ergodic rate from the $k$ th user in the $l$ th cell to its BS can be expressed as:

$$
\begin{aligned}
& \left\langle R_{l, k}\right\rangle \geq \log _{2}\left(1+p_{\mathrm{u}} \exp \left(\mathbb{E}_{X_{k}, Z_{l}}\left\{\ln \left(\frac{X_{k}}{p_{\mathrm{u}} Z_{l}+1}\right)\right\}\right)\right) \\
& =\log _{2}\left(1+p_{\mathrm{u}} \exp \left(\mathbb{E}_{X_{k}}\left\{\ln \left(X_{k}\right)\right\}-\mathbb{E}_{Z_{l}}\left\{\ln \left(p_{\mathrm{u}} Z_{l}+1\right)\right\}\right)\right)
\end{aligned}
$$

where we have exploited the fact that $\log _{2}(1+\alpha \exp (x))$ is convex in $x$ for $\alpha>0$ along with Jensen's inequality. We can now evaluate the expectations in (44) and we begin with $\mathbb{E}_{X_{k}}\left\{\ln \left(X_{k}\right)\right\}$, which can be expressed as

$$
\begin{aligned}
\mathbb{E}_{X_{k}}\left\{\ln \left(X_{k}\right)\right\} & =\frac{\beta_{l l k}^{-N+K-1}}{(N-K) !} \int_{0}^{\infty} \ln (x) e^{-x / \beta_{l l k}} x^{N-K} d x \\
& =\psi(N-K+1)+\ln \left(\beta_{l l k}\right)
\end{aligned}
$$

where we have used [34, Eq. (4.352.1)] to evaluate the corresponding integral. The second expectation in (44) requires a different line of reasoning. In particular, we have that

$$
\begin{aligned}
\mathbb{E}_{Z_{l}}\left\{\ln \left(p_{\mathrm{u}} Z_{l}+1\right)\right\} & =\sum_{\sum_{m=1}^{\varrho\left(\mathcal{A}_{l}\right)} \sum_{n=1}^{\tau_{m}\left(\mathcal{A}_{l}\right)} \mathcal{X}_{m, n}\left(\mathcal{A}_{l}\right) \frac{\mu_{l, m}^{-n}}{(n-1) !}} \underbrace{\int_{0}^{\infty} \ln \left(p_{\mathrm{u}} z+1\right) z^{n-1} e^{\frac{-z}{\mu_{l, m}}} d z}_{\triangleq \mathcal{I}} .
\end{aligned}
$$

The integral $\mathcal{I}$ admits the following manipulations

$$
\begin{aligned}
& \mathcal{I}=\int_{0}^{\infty} G_{2,2}^{1,2}\left[p_{\mathrm{u}} z \mid \begin{array}{l}
1,1 \\
1,0
\end{array}\right] z^{n-1} e^{\frac{-z}{\mu_{l, m}}} d z \\
& =\mu_{l, m}^{n} G_{3,2}^{1,3}\left[\begin{array}{l|l}
p_{\mathrm{u}} \mu_{l, m} & \begin{array}{c}
1-n, 1,1 \\
1,0
\end{array}
\end{array}\right]
\end{aligned}
$$

where $G_{p, q}^{m, n}\left[x, \mid \begin{array}{l}\alpha_{1}, \ldots, \alpha_{p} \\ \beta_{1}, \ldots, \beta_{q}\end{array}\right]$ denotes the Meijer's- $G$ function [34, Eq. (9.301)], and we have expressed the integrand $\ln (1+\alpha z)$ in terms of Meijer's- $G$ function according to [38, Eq. (8.4.6.5)]. The final expression stems from [34, Eq. (7.813.1)]. In addition, we can simplify (47) as follows

$$
\begin{aligned}
\mathcal{I} & =p_{\mathrm{u}} \mu_{l, m}^{n+1} G_{3,2}^{1,3}\left[p_{\mathrm{u}} \mu_{l, m} \mid \begin{array}{c}
-n, 0,0 \\
0,-1
\end{array}\right] \\
& =p_{\mathrm{u}} \mu_{l, m}^{n+1} \Gamma(n+1)_{3} F_{1}\left(n+1,1,1 ; 2 ;-p_{\mathrm{u}} \mu_{l, m}\right)
\end{aligned}
$$

where we have used [34, Eq. (9.31.5)] to obtain the first equality and [38, Eq. (8.4.51.1)] to obtain the second equality. Combining (46) with (48) and after some basic simplifications, we get

$$
\begin{aligned}
& \mathbb{E}_{Z_{l}}\left\{\ln \left(p_{\mathrm{u}} Z_{l}+1\right)\right\}=p_{\mathrm{u}} \sum_{m=1}^{\varrho\left(\mathcal{A}_{l}\right)} \sum_{n=1}^{\tau_{m}\left(\mathcal{A}_{l}\right)} \mu_{l, m} n \mathcal{X}_{m, n}\left(\mathcal{A}_{l}\right) \\
& \times{ }_{3} F_{1}\left(n+1,1,1 ; 2 ;-p_{\mathrm{u}} \mu_{l, m}\right) .
\end{aligned}
$$

Substituting (45) and (49) into (44), we conclude the proof.

\section{B. Proof of Proposition 4}

The MGF of $\gamma_{k}$ is given by

$$
\mathcal{M}_{\gamma_{k}}(s)=\mathbb{E}_{\gamma_{k}}\left\{e^{-s \gamma_{k}}\right\}=\int_{0}^{\infty} \mathbb{E}_{X_{k}}\left\{e^{-s \gamma_{k}}\right\} p_{Z_{l}}(z) d z \text {. }
$$

Using the PDF of $X_{k}$ given by (7), we have that

$$
\begin{aligned}
\mathbb{E}_{X_{k}}\left\{e^{-s \gamma_{k}}\right\} & =\frac{1}{(N-K) ! \beta_{l l k}^{N-K+1}} \\
& \times \int_{0}^{\infty} x^{N-K} \exp \left(-x\left(\frac{1}{\beta_{l l k}}+\frac{s}{z+1 / p_{\mathrm{u}}}\right)\right) d x \\
& =\left(\frac{z+1 / p_{\mathrm{u}}}{z+1 / p_{\mathrm{u}}+\beta_{l l k} s}\right)^{N-K+1}
\end{aligned}
$$


where the last equality is obtained by using [34, Eq. (3.326.2)]. Substituting (51) into (50) and using (8), we get

$$
\begin{aligned}
& \mathcal{M}_{\gamma_{k}}(s)=\int_{0}^{\infty} \sum_{m=1}^{\varrho\left(\mathcal{A}_{l}\right)} \sum_{n=1}^{\tau_{m}\left(\mathcal{A}_{l}\right)} \mathcal{X}_{m, n}\left(\mathcal{A}_{l}\right) \frac{\mu_{l, m}^{-n}}{(n-1) !} z^{n-1} e^{\frac{-z}{\mu_{l, m}}} \\
& \times\left(\frac{z+1 / p_{\mathrm{u}}}{z+1 / p_{\mathrm{u}}+\beta_{l l k} s}\right)^{N-K+1} d z \\
& =\sum_{m=1}^{\varrho\left(\mathcal{A}_{l}\right)} \sum_{n=1}^{\tau_{m}\left(\mathcal{A}_{l}\right)} \sum_{p=0}^{N-K+1}\left(\begin{array}{c}
N-K+1 \\
p
\end{array}\right) \mathcal{X}_{m, n}\left(\mathcal{A}_{l}\right) \frac{(-1)^{p} \mu_{l, m}^{-n}}{(n-1) !} \\
& \times\left(\frac{\beta_{l l k} s}{\beta_{l l k} s+1 / p_{\mathrm{u}}}\right)^{p} \int_{0}^{\infty} z^{n-1} e^{\frac{-z}{\mu l, m}}\left(\frac{z}{1 / p_{\mathrm{u}}+\beta_{l l k} s}+1\right)^{-p} d z
\end{aligned}
$$

where the last equality is obtained by using the binomial expansion formula. To evaluate the integral in (52), we first express $\left(\frac{z}{1 / p_{\mathrm{u}}+\beta_{l l k} s}+1\right)^{-p}$ in terms of a Meijer's- $G$ function with the help of [38, Eq. (8.4.2.5)], and then using the identity [38, Eq. (2.24.3.1)] to obtain

$$
\begin{aligned}
& \mathcal{M}_{\gamma_{k}}(s)=\sum_{m=1}^{\varrho\left(\mathcal{A}_{l}\right)} \sum_{n=1}^{\tau_{m}\left(\mathcal{A}_{l}\right)} \sum_{p=0}^{M-K+1}\left(\begin{array}{c}
N-K+1 \\
p
\end{array}\right) \mathcal{X}_{m, n}\left(\mathcal{A}_{l}\right) \frac{(-1)^{p}}{(n-1) !} \\
& \times\left(\frac{\beta_{l l k} s}{\beta_{l l k} s+1 / p_{\mathrm{u}}}\right)^{p} \frac{1}{\Gamma(p)} G_{2,1}^{1,2}\left[\left.\frac{\mu_{l, m}}{\beta_{l l k} s+1 / p_{\mathrm{u}}}\right|^{1-n, 1-p} \begin{array}{c}
0 \\
0
\end{array}\right] .
\end{aligned}
$$

Finally, using [38, Eq. (8.4.51.1)], we arrive at the desired result (19).

\section{Proof of Proposition 5}

From Proposition 1 and (26), we have

$$
P_{\text {out }} \triangleq \operatorname{Pr}\left(\frac{X_{k}}{Z_{l}+1 / p_{\mathrm{u}}} \leq \gamma_{\text {th }}\right)
$$

We can now express the above probability in integral form as follows:

$$
P_{\text {out }}=\int_{0}^{\infty} \operatorname{Pr}\left(X_{k}<\gamma_{\mathrm{th}}\left(Z_{\ell}+1 / p_{\mathrm{u}}\right) \mid Z_{\ell}\right) p_{Z_{\ell}}(z) d z
$$

The cumulative density function (CDF) of $X_{k}$ can be shown to be equal to

$$
F_{X_{k}}(x)=1-\exp \left(-\frac{x}{\beta_{l l k}}\right) \sum_{p=0}^{M-K} \frac{1}{p !}\left(\frac{x}{\beta_{l l k}}\right)^{p}
$$

where we have used the integral identity [34, Eq. (3.351.1)] to evaluate the CDF. Combining (55) with (56), we can rewrite $P_{\text {out }}$ as follows:

$$
\begin{aligned}
& P_{\text {out }}=1-\exp \left(-\frac{\gamma_{\mathrm{th}}}{p_{\mathrm{u}} \beta_{l l k}}\right) \sum_{p=0}^{N-K} \frac{\left(\frac{\gamma_{\mathrm{th}}}{\beta_{l l k}}\right)^{p}}{p !} \\
& \times \int_{0}^{\infty} \exp \left(-\frac{\gamma_{\mathrm{th}} z}{\beta_{l l k}}\right)\left(1 / p_{\mathrm{u}}+z\right)^{p} p_{Z_{\ell}}(z) d z \\
& =1-\exp \left(-\frac{\gamma_{\mathrm{th}}}{p_{\mathrm{u}} \beta_{l l k}}\right) \sum_{m=1}^{\varrho\left(\mathcal{A}_{l}\right)} \sum_{n=1}^{\tau_{m}\left(\mathcal{A}_{l}\right)} \sum_{p=0}^{N-K} \frac{\left(\frac{\gamma_{\mathrm{th}}}{\beta_{l l k}}\right)^{p}}{p !} \mathcal{X}_{m, n}\left(\mathcal{A}_{l}\right) \\
& \times \frac{\mu_{l, m}^{-n}}{(n-1) !} \int_{0}^{\infty}\left(\frac{1}{p_{\mathrm{u}}}+z\right)^{p} z^{n-1} \exp \left(-z\left(\frac{1}{\mu_{l, m}}+\frac{\gamma_{\mathrm{th}}}{\beta_{l l k}}\right)\right) d z .
\end{aligned}
$$

Applying a binomial expansion on (57) and thereafter evaluating the resulting integral using [34, Eq. (3.326.2)], we arrive at the desired result (27).

\section{REFERENCES}

[1] H. Q. Ngo, T. Q. Duong, and E. G. Larsson, "Uplink performance analysis of multicell MU-MIMO with zero-forcing receivers and perfect CSI," in Proc. IEEE Swedish Commun. Techn. Work. (Swe-CTW), Stockholm, Sweden, Nov. 2011, pp. 40-45.

[2] D. Gesbert, M. Kountouris, R. W. Heath Jr., C.-B. Chae, and T. Sälzer, "Shifting the MIMO paradigm," IEEE Signal Process. Mag., vol. 24, no. 5, pp. 36-46, Sept. 2007.

[3] M. M. da Silva, A. M. C. Correia, R. Dinis, N. Souto, and J. C. Silva, Transmission Techniques for $4 G$ Systems, 1st ed. Boca Raton, USA: CRC Press, 2012.

[4] L.-U. Choi and R. D. Murch, "A transmit preprocessing technique for multiuser MIMO systems using a decomposition approach," IEEE Trans. Wireless Commun., vol. 3, no. 1, pp. 20-24, Jan. 2004.

[5] N. Jindal and A. Goldsmith, "Dirty-paper coding versus TDMA for MIMO broadcast channels," IEEE Trans. Inf. Theory, vol. 51, no. 5, pp. 1783-1794, May 2005.

[6] P. Vishwanath and D. N. C. Tse, "Sum capacity of the vector Gaussian broadcast channel and uplink-downlink duality," IEEE Trans. Inf. Theory, vol. 49, no. 8, pp. 1912-1921, Aug. 2003.

[7] S. Catreux, P. F. Driessen, and L. J. Greenstein, "Simulation results for an interference-limited multiple-input multiple-output cellular system," IEEE Commun. Lett., vol. 4, no. 11, pp. 334-336, Feb. 2000.

[8] R. S. Blum, "MIMO capacity with interference," IEEE J. Sel. Areas Commun., vol. 21, no. 5, pp. 798-801, Nov. 2003.

[9] H. Dai, A. F. Molisch, and H. V. Poor, "Downlink capacity of interference-limited MIMO systems with joint detection," IEEE Trans. Wireless Commun., vol. 3, no. 2, pp. 798-801, Mar. 2004.

[10] D. Gesbert, S. V. Hanly, H. Huang, S. Shamai (Shitz), O. Simeone, and W. Yu, "Multi-cell MIMO cooperative networks: A new look at interference," IEEE J. Sel. Areas Commun., vol. 28, no. 9, pp. 13801408, Dec. 2010.

[11] J. Jose, A. Ashikhmin, T. L. Marzetta, and S. Vishwanath, "Pilot contamination and precoding in multi-cell TDD systems," IEEE Trans. Wireless Commun., vol. 10, no. 8, pp. 2640-2651, Aug. 2011.

[12] S. Verdú, Multiuser Detection. Cambridge, UK: Cambridge University Press, 1998.

[13] W. Choi and J. G. Andrews, "The capacity gain from intercell scheduling in multi-antenna systems," IEEE Trans. Wireless Commun., vol. 7, no. 2, pp. 714-725, Feb. 2008.

[14] C. Suh, M. Ho, and D. N. C. Tse, "Downlink interference alignment," IEEE Trans. Commun., vol. 59, no. 9, pp. 2616-2626, Sept. 2011.

[15] M. Matthaiou, N. D. Chatzidiamantis, G. K. Karagiannidis, and J. A. Nossek, "ZF detectors over correlated $K$ fading MIMO channels," IEEE Trans. Commun., vol. 59, no. 6, pp. 1591-1603, June 2011.

[16] C.-J. Chen and L.-C. Wang, "Performance analysis of scheduling in multiuser MIMO systems with zero-forcing receivers," IEEE J. Sel. Areas Commun., vol. 25, no. 7, pp. 1435-1445, Sept. 2007.

[17] A. Forenza, M. R. McKay, A. Pandharipande, R. W. Heath Jr., and I. B Collings, "Adaptive MIMO transmission for exploiting the capacity of spatially correlated channels," IEEE Trans. Veh. Technol., vol. 56, no. 2, pp. 619-630, Mar. 2007.

[18] S. Shim, J. S. Kwak, R. W. Heath, Jr., and J. G. Andrews, "Block diagonalization for multi-user MIMO with other-cell interference," IEEE Trans. Wireless Commun., vol. 7, no. 7, pp. 2671-2681, July 2008.

[19] F. Rusek, D. Persson, B. K. Lau, E. G. Larsson, T. L. Marzetta, O. Edfors, and F. Tufvesson, "Scaling up MIMO: Opportunities and challenges with very large arrays," IEEE Signal Process. Mag., vol. 30, no. 1, pp. 40-60, Jan. 2013.

[20] H. Q. Ngo, E. G. Larsson, and T. L. Marzetta, "Energy and spectral efficiency of very large multiuser MIMO systems," IEEE Trans. Commun., vol. 61, no. 4, pp. 1436-1449, April 2013.

[21] T. L. Marzetta, "Noncooperative cellular wireless with unlimited numbers of base station antennas," IEEE Trans. Wireless Commun., vol. 9, no. 11 , pp. 3590-3600, Nov. 2010

[22] R. Couillet and M. Debbah, "Signal processing in large systems: A new paradigm," IEEE Signal Process. Mag., vol. 30, no. 1, pp. 24-39, Jan. 2013.

[23] J. Hoydis, S. ten Brink, and M. Debbah, "Massive MIMO in the UL/DL of cellular networks: How many antennas do we need?" IEEE J. Sel. Areas Commun., vol. 31, no. 2, pp. 160-171, Feb. 2013. 
[24] M. R. McKay, I. B. Collings, and A. M. Tulino, "Achievable sum rate of MIMO MMSE receivers: A general analytic framework," IEEE Trans. Inf. Theory, vol. 56, no. 1, pp. 396-410, Jan. 2010.

[25] D. N. C. Tse and P. Viswanath, Fundamentals of Wireless Communications. Cambridge, UK: Cambridge University Press, 2005.

[26] K. Barman and O. Dabeer, "Capacity of MIMO systems with asynchronous PAM," IEEE Trans. Commun., vol. 57, no. 11, pp. 3366-3375, Nov. 2009.

[27] M. K. Simon and M.-S. Alouini, Digital Communication over Fading Channels: A Unified Approach to Performance Analysis. New York: Wiley, 2000.

[28] X. Gao, O. Edfors, F. Rusek, and F. Tufvesson, "Linear pre-coding performance in measured very-large MIMO channels," in Proc. IEEE Veh. Technol. Conf. (VTC), San Francisco, CA, Sept. 2011.

[29] J. Hoydis, "Random matrix methods for advance communication systems," Ph.D. dissertation, SUPELEC, France, April 2012.

[30] S. C. Schwartz and Y. S. Yeh, "On the distribution function and moments of power sums with log-normal components," Bell Syst. Tech. J., vol. 61, no. 7, Sept. 1982.

[31] H. Shin and M. Z. Win, "MIMO diversity in the presence of double scattering," IEEE Trans. Inf. Theory, vol. 54, no. 7, pp. 2976-2996, July 2008.

[32] D. A. Gore, R. W. Heath Jr., and A. J. Paulraj, "Transmit selection in spatial multiplexing systems," IEEE Commun. Lett., vol. 6, no. 11, pp. 491-493, Nov. 2002.

[33] A. Bletsas, H. Shin, and M. Z. Win, "Cooperative communications with outage-optimal opportunistic relaying," IEEE Trans. Wireless Commun., vol. 6, no. 9, pp. 3450-3460, Sept. 2007.

[34] I. S. Gradshteyn and I. M. Ryzhik, Table of Integrals, Series, and Products, 7th ed. San Diego, Academic Press, 2007.

[35] Wolfram, "The Wolfram functions site." Available: http://functions.wolfram.com

[36] M. R. McKay, A. Zanella, I. B. Collings, and M. Chiani, "Error probability and SINR analysis of optimum combining in Rician fading," IEEE Trans. Commun., vol. 57, no. 3, pp. 676-687, Mar. 2009.

[37] M. Kang and M.-S. Alouini, "Capacity of MIMO Rician channels," IEEE Trans. Wireless Commun., vol. 5, no. 1, pp. 112-122, Jan. 2006.

[38] A. P. Prudnikov, Y. A. Brychkov, and O. I. Marichev, Integrals and Series, Volume 3: More Special Functions. New York: Gordon and Breach Science, 1990.

[39] H. Shin and J. H. Lee, "Capacity of multiple-antenna fading channels: Spatial fading correlation, double scattering, and keyhole," IEEE Trans. Inf. Theory, vol. 49, no. 10, pp. 2636-2647, Oct. 2003.

[40] A. D. Wyner, "Shannon-theoretic approach to a Gaussian cellular multiple-access channel," IEEE Trans. Inf. Theory, vol. 40, no. 6, pp. 1713-1727, Nov. 1994.

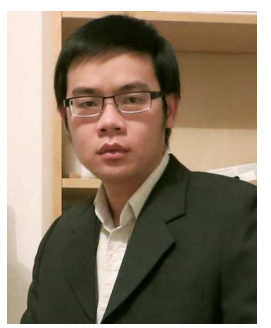

Hien Quoc Ngo received the B.S. degree in Electrical Engineering, major Telecommunications from Ho Chi Minh City University of Technology, Vietnam, in 2007, and the M.S. degree in Electronics and Radio Engineering from Kyung Hee University, Korea, in 2010. From 2008 to 2010, he was with the Communication and Coding Theory Laboratory, Kyung Hee University, where he did research on wireless communication and information theories, in particular cooperative communications, game theory and network connectivity. Since April 2010, he is a Ph.D. student of the Division for Communication Systems in the Department of Electrical Engineering (ISY) at Linköping University (LiU) in Linköping, Sweden. His current research interests include MIMO systems with very large antenna arrays (Massive MIMO), cooperative communications, and interference networks.

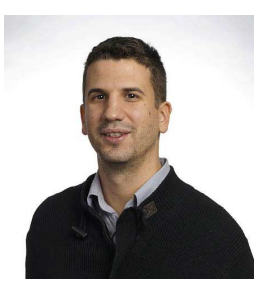

Michail Matthaiou (S'05-M'08) was born in Thessaloniki, Greece in 1981. He obtained the Diploma degree (5 years) in Electrical and Computer Engineering from the Aristotle University of Thessaloniki, Greece in 2004. He then received the M.Sc. (with distinction) in Communication Systems and Signal Processing from the University of Bristol, U.K. and Ph.D. degrees from the University of Edinburgh, U.K. in 2005 and 2008, respectively. From September 2008 through May 2010, he was with the Institute for Circuit Theory and Signal Processing, Munich University of Technology (TUM), Germany working as a Postdoctoral Research Associate. In June 2010, he joined Chalmers University of Technology, Sweden as an Assistant Professor and in 2011 he was awarded the Docent title. His research interests span signal processing for wireless communications, random matrix theory and multivariate statistics for MIMO systems, and performance analysis of fading channels.

Dr. Matthaiou is the recipient of the 2011 IEEE ComSoc Young Researcher Award for the Europe, Middle East and Africa Region and a co-recipient of the 2006 IEEE Communications Chapter Project Prize for the best M.Sc. dissertation in the area of communications. He was an Exemplary Reviewer for IEEE COMMUNICATIONS LETTERS for 2010. He has been a member of Technical Program Committees for several IEEE conferences such as ICC, GLOBECOM, etc. He currently serves as an Associate Editor for the IEEE TRANSACTIONS ON COMMUNICATIONS, IEEE COMMUNICATIONS LETTERS and was the Lead Guest Editor of the special issue on "Large-scale multiple antenna wireless systems" of the IEEE JOURNAL ON SELECTED AREAS IN COMMUNICATIONS. He is an associate member of the IEEE Signal Processing Society SPCOM and SAM technical committees.

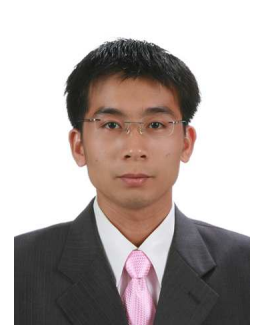

Trung Q. Duong (S'05-M'12-SM'13) was born in HoiAn town, Vietnam, in 1979. He received his $\mathrm{Ph} . \mathrm{D}$. degree in Telecommunications Systems from Blekinge Institute of Technology (BTH), Sweden in 2012. He has been working at BTH since 2008 and currently as a Project Manager. His current research interests include cross-layer design, cooperative communications, cognitive radio networks, physical layer security, and MIMO.

Dr. Duong has been a TPC chair for several international conferences and workshops, most recently in the IEEE GLOBECOM13 Workshop on Trusted Communications with Physical Layer Security. He currently serves as an Editor for the IEEE COMMUniCATIONS LetTERs, Wiley TRANSACTIONS ON EMERGING TELECOMMUNICATIONS TECHNOLOGIES and the Lead Guest Editor of the special issue on "Secure Physical Layer Communications" of the IET Communications, Guest Editor of the special issue on "Green Media: Toward Bringing the Gap between Wireless and Visual Networks" of the IEEE WIRELESS COMMUNICATIONS MAGAZINE, Guest Editor of the special issue on "Cooperative Cognitive Networks" of the EURASIP JOURNAL ON WIRELESS COMMUNICATIONS AND NETWORKING, Guest Editor of special issue on "Security Challenges and Issues in Cognitive Radio Networks" of the EURASIP Journal on AdVANCES Signal PROCESSING.

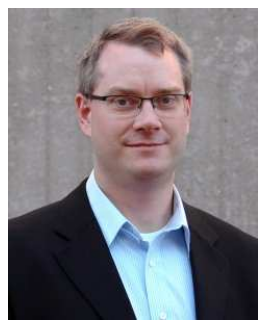

Erik G. Larsson received his Ph.D. degree from Uppsala University, Sweden, in 2002. Since 2007, he is Professor and Head of the Division for Communication Systems in the Department of Electrical Engineering (ISY) at Linköping University (LiU) in Linköping, Sweden. He has previously been Associate Professor (Docent) at the Royal Institute of Technology (KTH) in Stockholm, Sweden, and Assistant Professor at the University of Florida and the George Washington University, USA.

His main professional interests are within the areas of wireless communications and signal processing. He has published some 90 journal papers on these topics, he is co-author of the textbook SpaceTime Block Coding for Wireless Communications (Cambridge Univ. Press, 2003) and he holds 10 patents on wireless technology.

$\mathrm{He}$ is Associate Editor for the IEEE Transactions on Communications and he has previously been Associate Editor for several other IEEE journals. He is a member of the IEEE Signal Processing Society SPCOM technical committee. He is active in conference organization, most recently as the Technical Chair of the Asilomar Conference on Signals, Systems and Computers 2012 and Technical Program co-chair of the International Symposium on Turbo Codes and Iterative Information Processing 2012. He received the IEEE Signal Processing Magazine Best Column Award 2012. 